\title{
Residual limb volume change: Systematic review of measurement and
}

\section{management}

\author{
Joan E. Sanders, PhD; ${ }^{*}$ Stefania Fatone, $\mathbf{P h D}^{\mathbf{2}}$ \\ ${ }^{1}$ Department of Bioengineering, University of Washington, Seattle, WA; ${ }^{2}$ Northwestern University Prosthetics- \\ Orthotics Center, Department of Physical Medicine and Rehabilitation, Feinberg School of Medicine, Chicago, IL
}

\begin{abstract}
Management of residual limb volume affects decisions regarding timing of fit of the first prosthesis, when a new prosthetic socket is needed, design of a prosthetic socket, and prescription of accommodation strategies for daily volume fluctuations. This systematic review assesses what is known about measurement and management of residual limb volume change in persons with lower-limb amputation. Publications that met inclusion criteria were grouped into three categories: group I: descriptions of residual limb volume measurement techniques; group II: studies investigating the effect of residual limb volume change on clinical care in people with lower-limb amputation; and group III: studies of residual limb volume management techniques or descriptions of techniques for accommodating or controlling residual limb volume. We found that many techniques for the measurement of residual limb volume have been described but clinical use is limited largely because current techniques lack adequate resolution and insocket measurement capability. Overall, limited evidence exists regarding the management of residual limb volume, and the evidence available focuses primarily on adults with transtibial amputation in the early postoperative phase. While we can draw some insights from the available research about residual limb volume measurement and management, further research is required.
\end{abstract}

Key words: amputation, amputee, artificial limbs, measurement, prosthesis fitting, residual limb volume, shape, stump, suction, vacuum.

\section{INTRODUCTION}

\section{Scope of Problem}

Approximately 1 million persons in the United States live with lower-limb amputations [1], most of which ( 90\%) are the result of vascular disease [2]. Amputation rates are rising each year, in part because of the rapid increase in diabetes and also because of improvements in treatments for traumatic injury and vascular disease. More individuals live longer but require a limb amputation to survive. The recent wars in Iraq and Afghanistan have caused an increase in the number of service men and women who undergo an amputation [3-4], typically young individuals who were otherwise nondisabled at the time of their injury.

\footnotetext{
Abbreviations: $3-\mathrm{D}=$ three-dimensional, $\mathrm{AAOP}=$ American Academy of Orthotists and Prosthetists, CAD/CAM = computer-aided design/computer-aided manufacturing, $\mathrm{CI}=$ confidence interval, $\mathrm{CT}=$ computer tomography, ECF = extracellular fluid, EV = external validity, IV = internal validity, $\mathrm{MR}=$ magneto-rheologic, $\mathrm{MRI}=$ magnetic resonance imaging, $\mathrm{PVD}=$ peripheral vascular disease, $\mathrm{SD}=$ standard deviation, SEM = standard error of the mean, $\mathrm{SXCT}=$ spiral $\mathrm{X}$-ray computer tomography.

* Address all correspondence to Joan E. Sa nders, PhD; Department of Bio engineering, Box 355061, Foege N430J, 3720 15th Ave NE, University of Washington, Seattle, WA 98195-5061; 206-221-5872; fax: 206-616-2509.

Email: jsanders@u.washington.edu

DOI:10.1682/JRRD.2010.09.0189
} 
Regardless of etiology, the residual limb of adults with lower-limb amputation undergoes substantial change in shape and volume during the postoperative recovery period (defined as the first 12-18 months after amputation [5]). Even when "mature" (i.e., >18 months postamputation [5]), the residual limb will continue to experience daily fluctuations in volume, the magnitude of which vary among individuals [6-7] and may be problematic.

Changes in residual limb volume and shape lead to problems creating and maintaining an accurate and comfortable fit of the prosthetic socket. These problems are particularly severe for the immature residual limb (defined by Golbranson et al. [8] as $<12$ months postamputation) because edema and muscle atrophy occur postamputation. Thus, during the first few months postamputation, socket fit must be adjusted frequently. Edema control is particularly important for individuals with peripheral vascular disease (PVD) because impaired circulation due to swelling delays healing [9-10].

Inadequate control of residual limb volume postoperatively tends to delay prosthetic fitting. Advantages of early fitting with a prosthesis have been postulated to include accomplishing more normal gait reeducation, achieving a more independent life, undertaking more active physical training, gaining psychological advantages such as better acceptance of the amputation and restoration of body image, hastening maturation of the residual limb, and adapting the residual limb form to the definitive socket [11].

In the mature residual limb, both daily changes and long-term changes over weeks or months can occur. Shape and volume changes in the residual limb are believed important to changes in limb-socket interface pressure and shear stress distributions [12], which may in turn lead to socket fit problems, including gait instability and skin breakdown.

\section{Sources of Residual Limb Volume Change}

A prerequisite for total contact and good coupling (high stiffness) of the prosthetic socket/residual limb interface is a constant residual limb volume [13]. Residual limb volume change is caused by different sources, any of which may influence socket fit and function: generalized postoperative edema resulting from surgery and/ or injury to the limb [8,13-15]; postoperative muscle atrophy [8,13-14]; discrete, postoperative fluid collections quite distinct from generalized edema [16]; and residual limb muscle activity [13].
Edema is considered to be a pathological accumulation and retention of intracellular and extracellular fluid (ECF) in response to trauma and a main factor in prolonged amputee rehabilitation [17]. Edema decreases wound healing by limiting capillary blood supply [18]. Perfusion within the healing tissues may be increased through subjecting the residual limb to gravity by asking the patient to sit up or stand. Aside from requiring that patients be upright, ambulation may reduce edema by increasing counterpressure within the socket during weight-bearing and creating a muscular pumping effect that may help drive fluid out of the residual limb [19].

Diurnal volume changes in mature residual limbs are believed to be the result of three interrelated mechanisms: (1) pooling of blood in the venous compartment, (2) arterial vasodilatation, and (3) changes in the interstitial fluid volume [6-7]. Fluid movement in the residual limb is complex but in a basic sense is as follows: blood and plasma enter the residual limb through the arterial vasculature and exit through the venous vasculature. The interstitial fluid system, sometimes referred to as the lymphatic system, transports fluid through tissue external to the venous or arterial systems. Interstitial fluid emerges from the arterial vasculature and moves across arterial vessels into the interstitial space. Ultimately, it reenters the venous vasculature at some level. The velocity with which interstitial fluid moves is slow compared with arterial or venous blood flow velocities. However, this interstitial fluid is likely very important to prosthetic socket fit. When the volume of interstitial fluid moving from the arteries into the interstitial space is out of balance with that moving from the interstitial space into the lymphatics or veins, the residual limb will change volume [6].

\section{Postoperative Period}

The amputation and postoperative recovery period can be defined by five stages [5]: (1) the preoperative stage wherein the decision to amputate is made, vascular status is assessed, and level of amputation is selected; (2) the acute hospital postoperative phase (5-14 days postamputation); (3) the immediate postacute hospital stage (4-8 weeks postamputation), which is a time of recovery from surgery and early rehabilitation that typically ends when wounds are healed and the individual is ready for prosthetic fitting; (4) the intermediate recovery stage (4-6 months postamputation) wherein the individual transitions to his or her first formal prosthetic device with continuous adjustments required to accommodate rapid changes in 
residual limb volume that occur with the beginning of ambulation and prosthetic use; and (5) the transition to a stable stage (12-18 months postamputation), in which relative residual limb volume and shape stabilization occur.

Residual limb stabilization, wherein residual limb volume and shape are determined to be stable enough for a prosthesis to be used for an extended period of time, must occur before a definitive prosthetic fitting can take place. However, defining residual limb stabilization and time to first prosthetic fitting is very difficult.

\section{Postoperative Residual Limb Management Strategies}

During the immediate postacute hospital phase and when the individual is not wearing his or her prosthesis, wrapping and/or compression are used to help control residual limb volume and to shape the residual limb. The American Academy of Orthotists and Prosthetists (AAOP) State-of-the-Science Conference on Post-Operative Management of the Lower Extremity Amputee [5] identified five postoperative care strategies: (1) soft dressings, (2) nonremovable rigid dressings without an immediate prosthetic attachment, (3) nonremovable rigid dressings with an immediate postoperative prosthesis, (4) removable rigid plaster dressings, and (5) prefabricated postoperative prosthetic systems. Although some of these techniques may be applied immediately after surgery, this is not often done in practice [20]. Soft dressings such as elastic bandages, which are used most frequently for postoperative residual limb management, are normally not applied until adequate healing of the wound is achieved, generally around 21 days postamputation [21].

Once the initial (preparatory or interim) prosthesis has been fit, residual limb volume control strategies may include the use of liners, socks, pads, adjustable sockets, temporary sockets, or ambulatory check sockets [5]. While adding or removing material from the socket interface can help to reduce clinical problems arising from residual limb volume and shape changes, techniques such as adding socks to the residual limb add material to the interface uniformly. While sometimes these techniques are effective, residual limbs are unlikely to change volume and shape uniformly.

\section{Management of Mature Residual Limb Volume Fluctuations}

A residual limb may be considered to have reached maturity (i.e., relative stabilization of residual limb shape and volume) between 12 and 18 months postamputation
[5]. However, mature residual limbs are still subject to changes in volume, including diurnal fluctuations. The amount of daily volume fluctuation is likely to vary greatly among individuals with amputation as a function of comorbidities, prosthesis fit, activity level, ambient conditions, body composition, dietary habits, and for women, menstrual cycle. Current methods used to compensate for daily volume changes in the residual limb include addition and removal of socks, pads, inflatable air bladders, and fluid-filled bladders. Vacuum-assisted suspension systems are intended to indirectly hold residual limb soft tissues against the hard residual limb socket and have been suggested to retard limb volume reduction in part through improving fluid inflow into the residual limb so that it better balances with fluid outflow [22].

\section{Importance of Residual Limb Volume Issue}

Management of residual limb volume is important because it affects decisions regarding timing of fit of the first prosthesis, when a new prosthetic socket is needed, design of a prosthetic socket, and prescription of accommodation strategies for daily volume fluctuations. This review of residual limb volume literature is particularly timely with the increased number of socket volume accommodation technologies being pursued to adjust for diurnal residual limb volume change. Hence, the purpose of this systematic review was to assess what is known about measurement and management of residual limb volume change in persons with lower-limb amputation.

\section{METHODS}

We conducted a systematic review of the literature using several computer databases (PubMed 1950November 2009, Ovid MEDLINE 1966-November 2009, RECAL Legacy from the University of Strathclyde, and the Cochrane Database of Systematic Reviews 1991-November 2009). In addition, because the Journal of Prosthetics and Orthotics is not indexed by MEDLINE and is only current until December 2007 in RECAL Legacy, we searched it individually using “Journals@Ovid Full Text." The search terms used included "artificial limbs," "prosthesis fitting," "amputation stump," "volume," "measurement and shape," "suction," and "vacuum." References cited within publications identified by the search were also examined. 
Publications without abstracts, written in a language other than English, or published in trade magazines and newsletters were not considered. Publications were included if they described some aspect of a residual limb volume measurement technique, evaluated residual limb volume change in people with lower-limb amputation, or described a technique intended to accommodate residual limb volume change in people with lower-limb amputation. Publications were excluded if they did not provide an assessment of residual limb volume or did not use a model to estimate residual limb volume from measured data (e.g., circumference measurements). Inclusion was based on the consensus agreement between two reviewers (J.E.S and S.F.) who reviewed all abstracts. A total of 162 articles were initially identified, of which 52 met the inclusion criteria.

These publications were then grouped into the following categories: group I: descriptions of residual limb volume measurement techniques (Table 1); group II: studies investigating the effect of residual limb volume change on clinical care in people with lower-limb amputation; and group III: studies of residual limb volume management techniques in people with lower-limb amputation or descriptions of techniques for accommodating or controlling residual limb volume (Table 2). Group I was further subdivided into the measurement techniques described, group II into time postamputation, and group III into time postamputation and volume management technique. The Figure provides information as to the time postamputation of all studies reviewed in groups II and III.

Publications from group I were evaluated based on the performance of the described instrumentation and its sensitivity to the clinical challenges typically present when residual limb volume is measured in persons with amputation. Articles from groups II and III were initially reviewed using the AAOP Evidence Report Guidelines [23] (Appendix 1, available online only). The AAOP Quality Assessment Form [23] was then used to assess the internal validity (IV) and external validity (EV) (see Appendixes 2 and 3 for IV criteria IV-1 to IV-18 and Appendixes 4 and 5 for EV criteria EV-1 to EV-8, available online only). For this particular body of literature, fatigue and learning (IV-9) and accommodation and washout (IV-10) were applicable for only two studies [24-25]. For three articles classified as before and after trials (EV-5), criteria regarding group comparisons were not applicable (IV-2, IV-3, IV-4, and IV-12) [24-26].
Therefore, overall scores for IV/EV were assessed using the following criteria:

- If more than half of the applicable IV/EV variables were not addressed or poorly addressed (i.e., marked "no" or "unknown"), then the overall assessment was deemed to be low.

- If more than one but less than half of the applicable IV/EV variables were appropriately addressed (i.e., marked "yes"), then overall assessment was deemed to be moderate.

- If one or none of the applicable IV/EV variables were not addressed or poorly addressed (i.e., marked "no" or "unknown"), then the overall assessment was deemed to be high.

To be scored "yes" for EV-1 (sample characteristics adequately described), age, amputation level, time since amputation, and cause of amputation must have been described. If EV-1 was scored "no," then EV-2 (sample representative of target population) was also scored "no" because insufficient information was available to assess representativeness of the sample group. Appendix 6 provides a summary of the measurement errors for articles in groups II and III (available online only). Publications classified as "systematic review" or "expert opinion" were not assessed for quality.

\section{RESULTS OF REVIEW}

\section{Group I: What Techniques Have Been Used to Measure Residual Limb Volume and What Is Evidence that They Effectively Measure Residual Limb Volume in Persons with Amputation?}

\section{Measurement Techniques}

We identified 26 studies that described a number of methods to measure residual limb volume [15,27-51]. We summarize these methods here.

Water Displacement. The residual limb is placed within a cylinder filled to the brim with water. Water spills out of the cylinder as it is displaced by the volume of the residual limb. The mass of the displaced water or the difference in mass of water in the cylinder before and after limb insertion is used to calculate the volume of the residual limb. Segmental (sectional) limb volumes can be determined by monitoring the mass of displaced water while the limb is lowered into the water-filled cylinder [27] or, alternatively, while the cylinder is raised up on 
Table 1.

Summary of residual limb volume measurement techniques (group I articles).

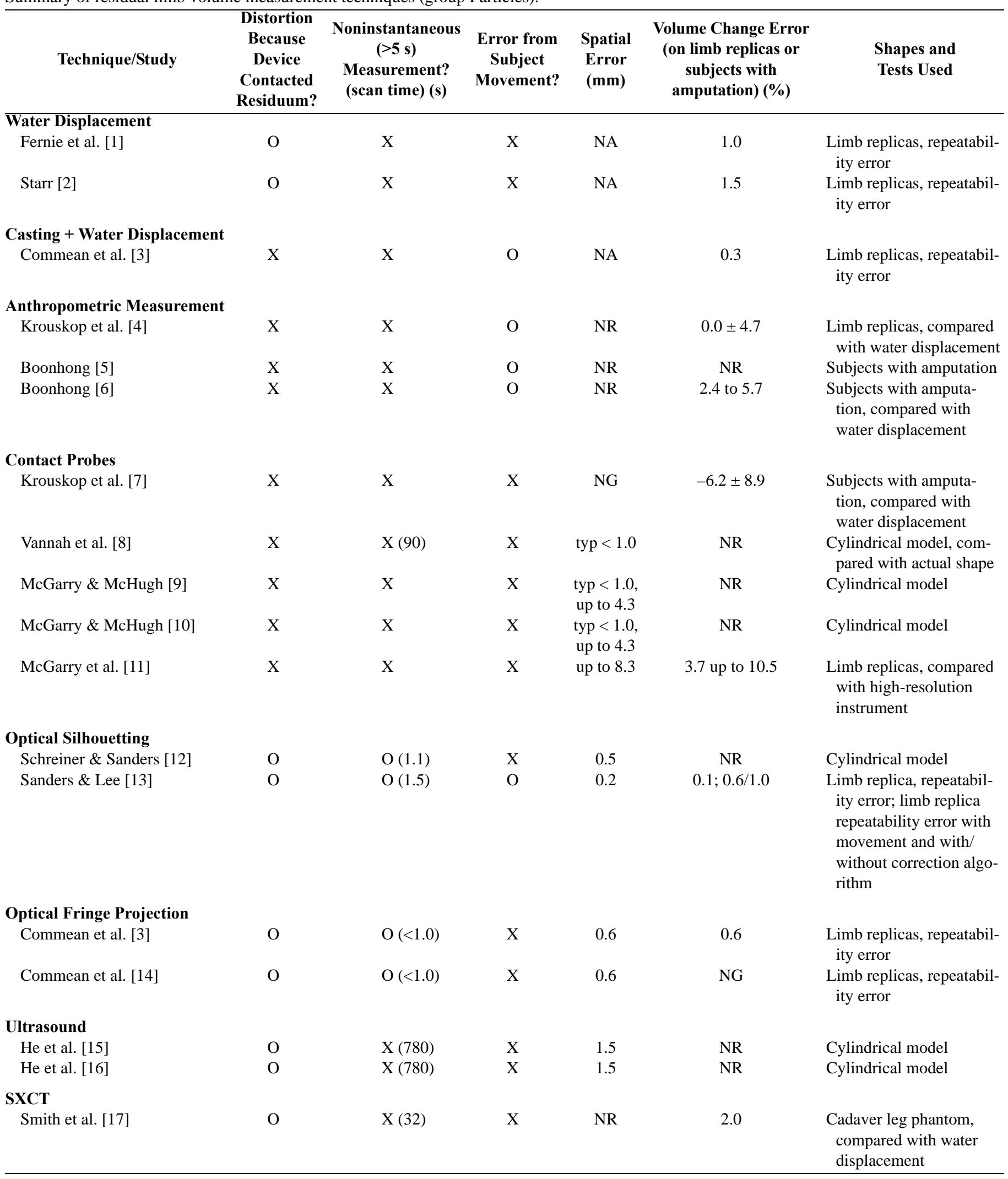


JRRD, Volume 48, Number 8, 2011

Table 1. (cont)

Summary of residual limb volume measurement techniques (group I articles).

\begin{tabular}{|c|c|c|c|c|c|c|}
\hline Technique/Study & $\begin{array}{l}\text { Distortion } \\
\text { Because } \\
\text { Device } \\
\text { Contacted } \\
\text { Residuum? }\end{array}$ & $\begin{array}{c}\text { Noninstantaneous } \\
\text { (>5 s) } \\
\text { Measurement? } \\
\text { (scan time) (s) }\end{array}$ & $\begin{array}{l}\text { Error from } \\
\text { Subject } \\
\text { Movement? }\end{array}$ & $\begin{array}{l}\text { Spatial } \\
\text { Error } \\
(\mathrm{mm})\end{array}$ & $\begin{array}{l}\text { Volume Change Error } \\
\text { (on limb replicas or } \\
\text { subjects with } \\
\text { amputation) }(\%)\end{array}$ & $\begin{array}{l}\text { Shapes and } \\
\text { Tests Used }\end{array}$ \\
\hline Smith et al. [18] & $\mathrm{O}$ & $\mathrm{X}(32)$ & $\mathrm{X}$ & NR & $<1.0$ & $\begin{array}{l}\text { Limb replicas, repeatabil- } \\
\text { ity error }\end{array}$ \\
\hline Smith et al. [19] & $\mathrm{O}$ & $X(32)$ & $\mathrm{X}$ & NR & $<1.0$ & $\begin{array}{l}\text { Subjects with amputa- } \\
\text { tion, repeatability error }\end{array}$ \\
\hline Commean et al. [20] & $\mathrm{O}$ & $X(32)$ & $\mathrm{O}$ & 1.0 & NG & $\begin{array}{l}\text { Markers placed on subjects } \\
\text { with amputation, repeat- } \\
\text { ability error }\end{array}$ \\
\hline
\end{tabular}

\section{Laser Scanning}

Fernie et al. [21]

Oberg et al. [22]

Lilja \& Oberg [23]

$\begin{array}{ll}\mathrm{O} & \mathrm{O}(0.6) \\ \mathrm{O} & \mathrm{X}(10) \\ \mathrm{O} & \mathrm{X}(10)\end{array}$

X NG

NG

$\begin{array}{llc}X & \text { NG } & \text { NG } \\ X & \text { NG } & 2.5 ; 0.5\end{array}$

No evaluation reported

No evaluation reported

23]

Johansson \& Oberg [24]

O X (10)

X NG

$0.3 ; 0.4$

Limb replicas, compared
with casting + water displacement; limb replicas, repeatability error Limb replicas, compared with casting + water displacement; limb replicas, repeatability error

\section{MRI}

Buis et al. [25]

O X (592)

O

0.6

NG

Subjects with amputation, compared with displacement gauge and MRI system resolution

\section{Bioimpedance}

Sanders et al. [26]

O $\quad \mathrm{O}(<1.0)$

O NA

0.2

Nondisabled subjects, repeatability during standing

1. Fernie GR, Holliday PJ, Lobb RJ. An instrument for monitoring stump oedema and shrinkage in amputees. Prosthet Orthot Int. 1978;2(2):69-72. [PMID: 364403] DOI:10.3109/03093647809177770

2. Starr TW. A computerized device for the volumetric analysis of the residual limbs of amputees. Bull Prosthet Res. 1980;10-33:98-102. [PMID: 7236952]

3. Commean PK, Smith KE, Cheverud JM, Vannier MW. Precision of surface measurements for below knee residua. Arch Phys Med Rehabil. 1996;77(5):477-86.

[PMID: 8629925]

DOI:10.1016/S0003-9993(96)90037-4

4. Krouskop TA, Yalcinkaya M, Muilenberg AL, Holland KC, Zuniga EN. A measurement technique to assess residual limb volume. Orthop Rev. 1979;8:69-77.

5. Boonhong J. Correlation between volumes and circumferences of residual limb in below knee amputees. J Med Assoc Thai. 2006;89(Suppl 3):S1-4. [PMID: 17722302]

6. Boonhong J. Validity and reliability of girth measurement (circumference measurement) for calculating residual limb volume in below-knee amputees. Chula Med J. 2007;51:77-88.

7. Krouskop TA, Dougherty D, Yalcinkaya MI, Muilenberg A. Measuring the shape and volume of an above-knee stump. Prosthet Orthot Int. 1988;12(3):136-42. [PMID: 3217243]

8. Vannah W, Drvaric DM, Stand JA, Hastings JA, Slocum JE, Harning DM, Gorton GE. Performance of a continuously sampling hand-held digitizer for residual-limb shape measurement. J Prosthet Orthot. 1997;9(4):157-62. DOI:10.1097/00008526-199700940-00006

9. McGarry T, McHugh B. Evaluation of a contemporary CAD/CAM system. Prosthet Orthot Int. 2005;29(3):221-29. [PMID: 16466152]

DOI:10.1080/03093640500199497

10. McGarry T, McHugh B. Comparison of the results of four users of a contemporary CAD/CAM system. Prosthet Orthot Int. 2007;31(1):27-35. [PMID: 17365882] DOI:10.1080/03093640600942101

11. McGarry T, McHugh B, Buis A, McKay G. Evaluation of the effect of shape on a contemporary CAD system. Prosthet Orthot Int. 2008;32(2):145-54. [PMID: 18569882] 
Table 1. (cont)

Summary of residual limb volume measurement techniques (group I articles).

12. Schreiner RE, Sanders JE. A silhouetting shape sensor for the residual limb of a below-knee amputee. IEEE Trans Rehabil Eng. 1995;3(3): 242-53. DOI:10.1109/86.413197

13. Sanders JE, Lee GS. A means to accommodate residual limb movement during optical scanning: A technical note. IEEE Trans Neural Sys Rehabil Eng. 2008;16(5):505-9. [PMID: 18990654] DOI:10.1109/TNSRE.2008.2003388

14. Commean PK, Smith KE, Vannier MW. Design of a 3-D surface scanner for lower limb prosthetics: A technical note. J Rehabil Res Dev. 1996;33(3):267-78. [PMID: 8823674]

15. He P, Xue K, Chen Q, Murka P, Schall S. A PC-based ultrasonic data acquisition system for computer-aided prosthetic socket design. IEEE Trans Rehabil Eng. 1996;4(2):114-19. [PMID: 8798078] DOI:10.1109/86.506408

16. He P, Xue K, Murka P. 3-D imaging of residual limbs using ultrasound. J Rehabil Res Dev. 1997;34(3):269-78. [PMID: 9239619]

17. Smith KE, Vannier MW, Commean PK. Spiral CT volumetry for below-knee residua. IEEE Trans Rehabil Eng. 1995;3(3):235-41. DOI:10.1109/86.413196

18. Smith KE, Commean PK, Bhatia G, Vannier MW. Validation of spiral CT and optical surface scanning for lower limb stump volumetry. Prosthet Orthot Int. 1995;19:97-107.

19. Smith KE, Commean PK, Vannier MW. Residual-limb shape change: Three-dimensional CT scan measurement and depiction in vivo. Radiology. 1996;200(3): 843-50. [PMID: 8756942]

20. Commean PK, Brunsden BS, Smith KE, Vannier MW. Below-knee residual limb shape change measurement and visualization. Arch Phys Med Rehabil. 1998; 79(7):772-82. [PMID: 9685090] DOI:10.1016/S0003-9993(98)90355-0

21. Fernie GR, Griggs G, Bartlett S, Lunau K. Shape sensing for computer aided below-knee prosthetic socket design. Prosthet Orthot Int. 1985;9(1):12-16. [PMID: 4000905]

22. Oberg K, Kofman J, Karisson A, Lindstrom B, Siglad G. The CAPOD system—A Scandinavian CAD/CAM system for prosthetic sockets. J Prosthet Orthot. 1989;1(3):139-48. DOI:10.1097/00008526-198904000-00008

23. Lilja M, Oberg T. Volumetric determinations with CAD/CAM in prosthetics and orthotics: Errors of measurement. J Rehabil Res Dev. 1995;32(2):141-48. [PMID: 7562654]

24. Johansson S, Oberg T. Accuracy and precision of volumetric determinations using two commercial CAD systems for prosthetics: A technical note. J Rehabil Res Dev. 1998;35(1):27-33. [PMID: 9505250]

25. Buis AW, Condon B, Brennan D, McHugh B, Hadley D. Magnetic resonance imaging technology in transtibial socket research: A pilot study. J Rehabil Res Dev. 2006;43(7):883-90. [PMID: 17436174] DOI:10.1682/JRRD.2005.08.0145

26. Sanders JE, Rogers EL, Abrahamson DC. Assessment of residual-limb volume change using bioimpedence. J Rehabil Res Dev. 2007;44(4):525-36. [PMID: 18247249] DOI:10.1682/JRRD.2006.08.0096

MRI = magnetic resonance imaging, $\mathrm{NA}=$ not applicable, $\mathrm{NG}=$ not given, $\mathrm{NR}=$ not relevant because testing was conducted on cylindrical model, $\mathrm{O}=$ not present, $\underline{\text { SXCT }=\text { spiral X-ray computer tomography, typ = typically, } \mathrm{X}=\text { present }}$

the limb [28]. However, the measurement is sensitive to surface tension effects at the limb-water interface and care must be taken to ensure that surface tension does not distort the measurement of interest.

Fernie et al., measuring a plexiglass model of a transfemoral residual limb 20 times, reported that measurements were repeatable within $\sim 1.0$ percent [27]. Starr reported similar results of 1.3 percent [28]. Starr noted, however, that measurement error increased to between 2.1 and 3.7 percent when residual limbs were measured, because of subject tremor and vertical motion during the measurement process (approximately 10 seconds duration).

Casting + Water Displacement. A variation of the water displacement technique, and one that avoids the surface tension problem, is to first take a cast of the residual limb, with the practitioner applying minimal tension to the bandage during wrapping, or, alternatively, to take a cast using an alginate casting technique. A positive model is then made from the cast and its volume is measured using the water displacement technique described in the previous section. A similar technique was used by Commean et al., except that they made a positive model from the cast and then scanned the model [29]. Repeatability error for the casting + water displacement technique was reported to be $<1.0$ percent. The technique was also used by Johansson and Oberg to evaluate the volumes of sockets manufactured using computer-aided design/computer-aided manufacturing (CAD/CAM) methods [49].

Anthropometric Measurements. A tape measure or calipers are used to measure distances between anatomical landmarks on the residual limb and/or its circumference: those measurements are then used in an anthropometric model to determine residual limb volume [30-31]. Krouskop et al. measured circumferences at $5 \mathrm{~cm}$ intervals down the lengths of 16 representative transfemoral amputation plaster casts and found that conical frustrum model 
JRRD, Volume 48, Number 8, 2011

Table 2.

Summary of articles included in groups II and III.

\begin{tabular}{|c|c|c|c|c|c|c|}
\hline Author & Group & Measurement Method & $\begin{array}{c}\text { Study } \\
\text { Design* }\end{array}$ & Internal Validity & $\begin{array}{l}\text { External } \\
\text { Validity }\end{array}$ & Additional Category \\
\hline Goldberg et al. [1] & III & Water Displacement & E5 & Low & Low & Early limbs, whirlpool therapy \\
\hline Manella [2] & III & Anthropometric & E1 & Moderate & High & $\begin{array}{l}\text { Early limbs, shrinker sock vs } \\
\text { EB }\end{array}$ \\
\hline Fernie \& Holliday [3] & II & Water Displacement & O1 & Moderate & Moderate & Early and mature limbs \\
\hline Mueller [4] & III & Anthropometric & E1 & Moderate & Moderate & Early limbs, RRD vs EB \\
\hline Liedberg et al. [5] & II \& III & Anthropometric & E1 & Low & Low & Early limbs, adaptable device \\
\hline Persson \& Liedberg [6] & II & Anthropometric & O5 & Low & Moderate & Early limbs \\
\hline Wilson et al. [7] & III & NA & $\mathrm{X} 2$ & NA & NA & Adaptable device \\
\hline Golbranson et al. [8] & II & $\begin{array}{l}\text { Anthropometric Water } \\
\text { Displacement }\end{array}$ & E2 & Low & Moderate & $\begin{array}{l}\text { Correlated measurement tech- } \\
\text { niques in early limbs }\end{array}$ \\
\hline Pinzur et al. [9] & III & NA & $\mathrm{X} 2$ & NA & NA & Adaptable device \\
\hline MacLean \& Fick [10] & II \& III & Anthropometric & E2 & Moderate & High & Early limbs, SRD vs EB \\
\hline Lilja \& Oberg [11] & II & Laser Scan & O5 & Low & High & Early limbs \\
\hline Lilja et al. [12] & II & MRI & O6 & Low & High & Early limbs \\
\hline Lilja et al. [13] & II & Laser Scan & O5 & Moderate & Moderate & Early limbs \\
\hline Wong \& Edelstein [14] & II \& III & Anthropometric & E1 & Moderate & High & Early limbs, SRD vs EB \\
\hline Board et al. [15] & III & $\begin{array}{l}\text { Casting + Water Dis- } \\
\text { placement }\end{array}$ & E5 & Low & Moderate & $\begin{array}{l}\text { Mature limbs, vacuum-assisted } \\
\text { suspension }\end{array}$ \\
\hline Goswami et al. [16] & III & $\begin{array}{l}\text { Casting + Water Dis- } \\
\text { placement }\end{array}$ & E5 & Low & Moderate & $\begin{array}{l}\text { Mature limbs, vacuum-assisted } \\
\text { suspension }\end{array}$ \\
\hline Graf \& Freijah [17] & III & $\begin{array}{l}\text { Casting + Water Fill/ } \\
\text { Displacement }\end{array}$ & E1 & Moderate & High & $\begin{array}{l}\text { Early limbs, RRD vs RRD + gel } \\
\text { sock }\end{array}$ \\
\hline Greenwald et al. [18] & III & Fluid-Filled Bladders & O6 & Low & Low & Adaptable device \\
\hline Zachariah et al. [19] & II & Optical Scan & O5 & High & High & Mature limbs \\
\hline Nawijn et al. [20] & III & NA & S2 & NA & NA & Systematic review \\
\hline Sanders et al. [21] & II & Optical Scan & O5 & Moderate & High & Mature limbs \\
\hline Sanders et al. [22] & III & Fluid-Filled Bladders & O6 & High & High & Adaptable device \\
\hline Singh et al. [23] & II & Ultrasound & $\mathrm{O} 1$ & Moderate & High & Early limbs \\
\hline Janchai et al. [24] & III & Anthropometric & E1 & Moderate & High & Early limbs, RRD vs EB \\
\hline Ogawa et al. [25] & III & Fluid-Filled Bladders & O6 & Low & Low & Adaptable device \\
\hline Sanders et al. [26] & I \& II & Bioimpedance & O5 & High & High & Mature limbs \\
\hline
\end{tabular}

*Refer to Appendix 1 (available online only) and Hafner [27] for study design descriptors.

1. Goldberg MJ, Culver JV, Carson JF. Volume changes in below-knee amputation stumps as affected by type of whirpool-tank hydrotherapy. J Am Gerontol Soc. 1968;16(1):101-105. [PMID: 5634466]

2. Manella KJ. Comparing the effectiveness of elastic bandages and shrinker socks for lower extremity amputees. Phys Ther. 1981;61(3):334-37. [PMID: 7465627

3. Fernie GR, Holliday PJ. Volume fluctuations in the residual limbs of lower limb amputees. Arch Phys Med Rehabil. 1982;63(4):162-65. [PMID: 7082139]

4. Mueller MJ. Comparison of removable rigid dressings and elastic bandages in preprosthetic management of patients with below-knee amputations. Phys Ther. 1982;62(10):1438-41. [PMID: 7122702]

5. Liedberg E, Hommerberg H, Persson BM. Tolerance of early walking with total contact among below-knee amputees-A randomized test. Prosthet Orthot Int. 1983;7(2):91-95. [PMID: 6622240]

6. Persson BM, Liedberg E. A clinical standard of stump measurement and classification in lower limb amputees. Prosthet Orthot Int. 1983;7(1):17-24. [PMID: 6856447]

7. Wilson AB Jr, Schuch CM, Nitschke RO. A variable volume socket for below-knee prostheses. Clin Prosthet Orthot. 1987;11(1):11-19.

8. Golbranson FL, Wirta RW, Kuncir EJ, Lieber RL, Oishi C. Volume changes occurring in postoperative below-knee residual limbs. J Rehabil Res Dev. 1988;25(2):11-18. [PMID: 3361456]

9. Pinzur MS, Angelico JA, Quigley MJ. A volume-adaptable prosthesis for ankle disarticulation. J Prosthet Orthot. 1993;5:77-78. DOI:10.1097/00008526-199307000-00003

10. MacLean N, Fick GH. The effect of semirigid dressings on below-knee amputations. Phys Ther. 1994;74(7):668-73. [PMID: 8016199]

11. Lilja M, Oberg T. Proper time for definitive transtibial prosthetic fitting. J Prosthet Orthot. 1997;9(2):90-95. DOI:10.1097/00008526-199700920-00009

12. Lilja M, Hoffmann P, Oberg T. Morphological changes during early trans-tibial prosthetic fitting. Prosthet Orthot Int. 1998;22(2):115-22. [PMID: 9747995]

13. Lilja M, Johansson S, Oberg T. Relaxed versus activated stump muscles during casting for trans-tibial prostheses. Prosthet Orthot Int. 1999;23(1):13-20. [PMID: 10355639]

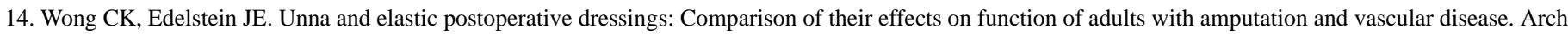
Phys Med Rehabil. 2000;81(9):1191-98. [PMID: 10987161] DOI:10.1053/apmr.2000.3780 
Table 2. (cont)

Summary of articles included in groups II and III.

15. Board WJ, Street GM, Caspers C. A comparison of trans-tibial amputee suction and vacuum socket conditions. Prosthet Orthot Int. 2001;25(3):202-9. [PMID: 11860094] DOI:10.1080/03093640108726603

16. Goswami J, Lynn R, Street G, Harlander M. Walking in a vacuum-assisted socket shifts the stump fluid balance. Prosthet Orthot Int. 2003;27(2):107-13. [PMID: 14571940] DOI:10.1080/03093640308726666

17. Graf M, Freijah N. Early trans-tibial oedema control using polymer gel socks. Prosthet Orthot Int. 2003;27(3):221-26. [PMID: 14727703] DOI:10.1080/03093640308726685

18. Greenwald RM, Dean RC, Board WJ. Volume management: Smart Variable Geometry Socket (SVGS) technology for lower-limb prostheses. J Prosthet Orthot. 2003;15(3):107-12. DOI:10.1097/00008526-200307000-00011

19. Zachariah SC, Saxena R, Fergason JR, Sanders JE. Shape and volume change in the transtibial residuum over the short term: Preliminary investigation of six subjects. J Rehabil Res Dev. 2004;41(5):683-94. [PMID: 15558398] DOI:10.1682/JRRD.2003.10.0153

20. Nawijn SE, Van der Linde H, Emmelot CH, Hofstad CJ. Stump management after trans-tibial amputation: A systematic review. Prosthet Orthot Int. 2005;29(1): 13-26. [PMID: 16180374] DOI:10.1080/17461550500066832

21. Sanders JE, Zachariah SG, Jacobsen AK, Fergason JR. Changes in interface pressures and shear stresses over time on trans-tibial amputee subjects ambulating with prosthetic limbs: Comparison of diurnal and six-month differences. J Biomech. 2005;38(8):1566-73. [PMID: 15958212] DOI:10.1016/j.jbiomech.2004.08.008

22. Sanders JE, Jacobsen AK, Fergason JR. Effects of fluid insert volume changes on socket pressures and shear stresses: Case studies from two trans-tibial amputee subjects. Prosthet Orthot Int. 2006;30(3):257-69. [PMID: 17162516] DOI:10.1080/03093640600810266

23. Singh R, Hunter J, Philip A. Fluid collections in amputee stumps: A common phenomenon. Arch Phys Med Rehabil. 2007;88(5):661-63. [PMID: 17466737] DOI:10.1016/j.apmr.2007.02.016

24. Janchai S, Boonhong J, Tiamprasit J. Comparison of removable rigid dressing and elastic bandage in reducing the residual limb volume of below knee amputees. J Med Assoc Thai. 2008;91(9):1441-46. [PMID: 18843876]

25. Ogawa A, Obinata G, Hase K, Dutta A, Nakagawa M. Design of lower limb prosthesis with contact pressure adjustment by MR fluid. Conf Proc IEEE Eng Med Biol Soc. 2008;2008:330-33. [PMID: 19162660]

26. Sanders JE, Harrison DS, Allyn KJ, Myers TR. Clinical utility of in-socket residual limb volume change measurement: Case study results. Prosthet Orthot Int. 2009;33(4):378-90. [PMID: 19961297] DOI:10.3109/03093640903214067

27. Hafner B. American Academy of Orthotists and Prosthetists state-of-the-science evidence reports. The Academy Today. 2008;4(2):A4-A8.

$\mathrm{EB}$ = elastic bandage, $\mathrm{MRI}=$ magnetic resonance imaging, $\mathrm{NA}=$ not applicable, $\mathrm{RRD}=$ removable rigid dressing, $\mathrm{SRD}=$ semirigid dressing.

volumes calculated from those measurements were within 8.1 percent of cast volumes measured using water displacement [30]. The mean difference was 0.0 percent, but the standard deviation (SD) was 4.7 percent. Boonhong [31] extended Krouskop et al.’s [30] effort, measuring circumference at 3 to 6 positions down the length of 51 transtibial residual limbs and then used both a conical frustrum model and a cylindrical model to calculate volumes. Boonhong found that although the anthropometric measurements tended to overestimate residual limb volume, the 95 percent confidence interval (CI) of the difference in mean volume measured by water displacement and anthropometric measurements was 4.0 percent of the mean volume measured by water displacement. The 95 percent CI for repeatability testing using circumferential measurements was $~ 1.1$ percent of the mean limb volume. Further, from measurements on 31 subjects with transtibial amputation of $<6.5$ months duration, Boonhong concluded that residual limb volumes calculated from circumference measurements were not reliable enough to accurately measure changes in limb volume over a 1-month period soon after amputation [31]. Mean volume changes 1 month apart averaged 5.8 percent, but the 95 percent CI for the measurement performed using circumference measurements was $>55$ percent of the mean volume change. Based on Boonhong's results, we estimate error in the measurement of limb volume change using his circumference measurement method to be between 2.4 and 5.7 percent.

Boonhong [15] and Golbranson et al. [8] attempted to determine whether residual limb circumference measurements were directly correlated with residual limb volume. If a strong relationship was demonstrated, then a cylindrical or frustrum model as described previously would not be needed and limb volume could be estimated directly from perhaps just a single circumference measurement. Limb circumferences were measured with a tape measure, and limb volume was measured using water displacement. Boonhong studied 55 persons with transtibial amputation (median age 56 years, range 43-67; median time postamputation 5.43 months, range 3.53 to 15.8 ; 


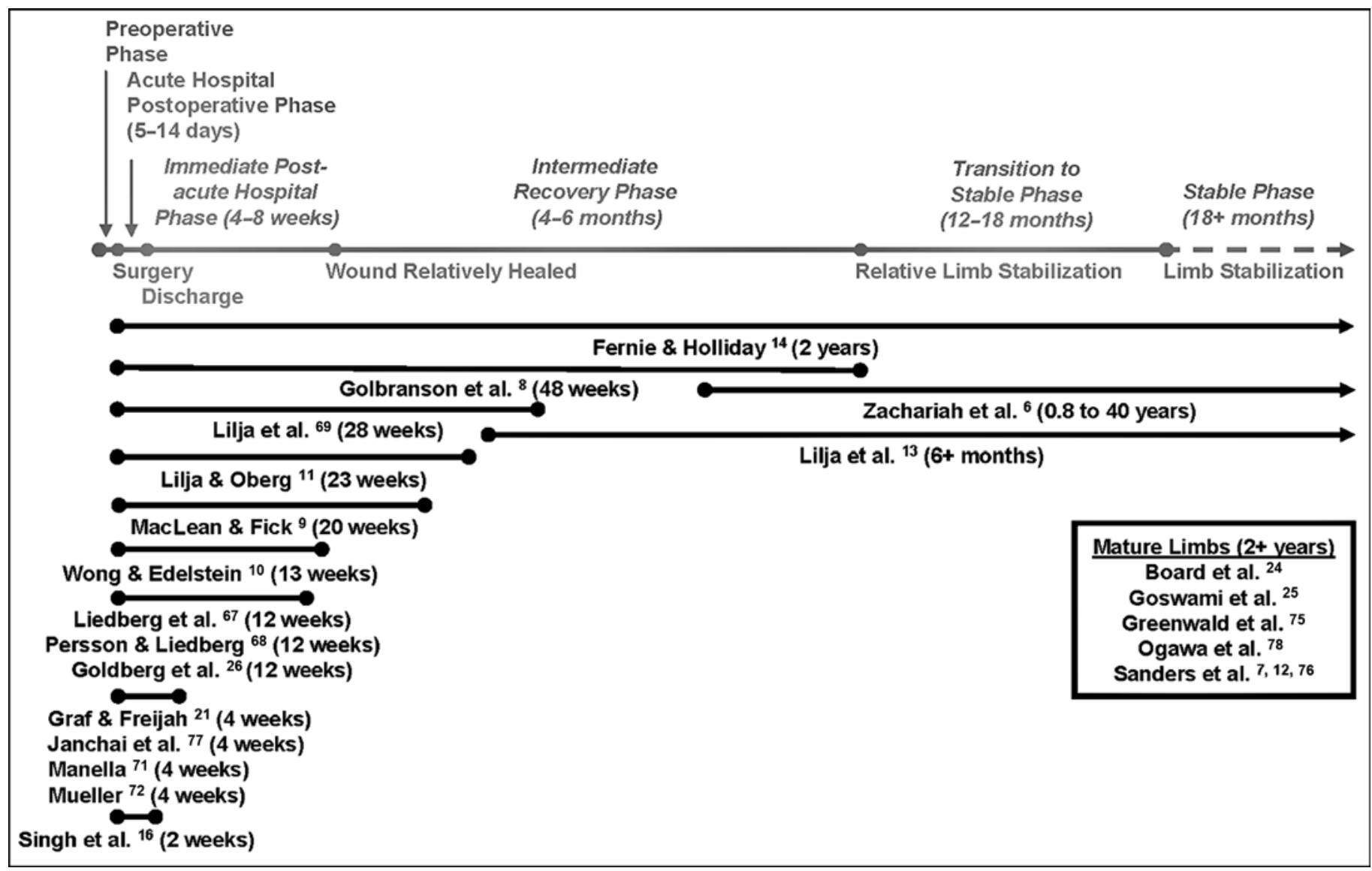

Figure.

Time postamputation of all studies reviewed in groups II and III shown in black text (except for Pinzur et al. [74] and Wilson et al. [73], who did not report data on people with lower-limb amputation, and Nawijn et al. [17], which was a review of multiple studies). For reference, gray text indicates postamputation phases taken from American Academy of Orthotists and Prosthetists report on postoperative management of persons with amputation [5]. Superscript numbers and reference numbers in caption (in brackets) refer to main article reference numbers. Majority of reviewed studies report on residual limb volume during immediate postacute hospital stage (4ᄀ8 weeks) and intermediate recovery stage (4-6 months).

mean \pm SD residual limb length $11.08 \pm 2.81 \mathrm{~cm}$, range 6-18) [15]. Residual limb circumferences were measured at four levels (tibial tubercle, $4 \mathrm{~cm}$ and $8 \mathrm{~cm}$ below the tibial tubercle, and distal end). Cause of amputation was not reported. A single person took all measurements twice in set order (circumferences first then residual limb volume). The author reported that residual limb volume and circumference were significantly correlated at all levels tested $(0.81,0.89,0.88$, and 0.72 , respectively). The results of this study were used to hypothesize that residual limb circumference measurements taken at $4 \mathrm{~cm}$ below the tibial tubercle could be used to track residual limb volume change over time. However, no measurements of limb volume change or circumference change were conducted to confirm this hypothesis, and in a subsequent investigation, Boonhong noted that a high correlation does not mean that two measurement methods agree with each other [31].

Golbranson et al. measured limb volume and circumference change on 36 subjects during 14 weeks postamputation [8], using tape measurements for limb circumference and water displacement for volume assessment similar to Boonhong's [15] measurement technique. Golbranson et al. reported good correlation between volume change and circumference change only when both parameters increased, which was not always the case in this cohort of subjects [8]. This led Golbranson et al. to conclude that measurements of circumference change were not reflective of limb volume change [8]. However, a large amount of limb volume fluctuation from session to 
session was noted in Golbranson et al.'s results [8], possibly reflecting error from inconsistent marker placement or subject movement during the water displacement measurement procedure, issues discussed more completely in "Sources of Error and Characteristics of Measurement Techniques" (p. 961). Based on Boonhong's [15] and Golbranson et al.'s [8] findings, a good correlation between residual limb circumference and residual limb volume may exist when both increase, but the accuracy of circumferential measurements for limb volume change measurement is unknown, and thus, its capability to measure volume fluctuations relevant to patient care is unknown.

Contact Probes. A small contact probe touches the surface of the residual limb, measuring the position of contact with the skin in space. An instrumented mechanical arm or an electromagnetic position sensor is typically used for position measurement. The probe is either passed over the residual limb manually by the user or automatically rotated around the limb by a machine [32-33]. Krouskop et al. rotated a stylus arm around transfemoral residual limbs, measuring radius at $5^{\circ}$ intervals in cross sections spaced at $2.5 \mathrm{~cm}$ increments down the limb length [32]. Their device produced volume measurements that were within -6.2 percent ( $\mathrm{SD}=8.9 \%$ ) of volumes measured using water displacement; thus on average, their contact probe volumes were less than water displacement volumes. Vannah et al. used a stylus mounted to an electromagnetic position sensor to digitize cylindrical plaster casts [33]. They found that radial measurement errors were typically $<1.0 \mathrm{~mm}$. McGarry et al. extended from Vannah et al.'s evaluation efforts and tested a commercial contact digitizer pen [34-36]. They found that performance results from transtibial limb replicas were worse than those from cylindrical models. Volume errors for limb replicas averaged 3.7 percent and ranged up to 10.5 percent. McGarry et al., citing Lilja et al. [13], noted that a 5 percent volume difference corresponds to approximately one terry toweling sock; thus volume errors using the contact digitizer pen were within a two-sock error range [36]. Geil reported that limb circumference measurements from a contact probe were comparable to those from a springloaded tape measure [52].

\section{Optical Scanning (Silhouetting; Fringe Projec-}

tion). Silhouetting $[37,43]$ or fringe projection $[29,39]$ are two methods used to perform optical scanning. With silhouetting, the outside contour of the residual limb is viewed from different angles. With fringe projection, a fringe or grid pattern is projected onto the residual limb and viewed from different angles. With both methods, digital images are taken from multiple positions around the limb and then the data are used to create a threedimensional (3-D) model of the residual limb surface from which limb volume is calculated. A strength of optical scanners is that they image very quickly. Schreiner and Sanders' scanner acquired images in 1.1 seconds [37], Sanders and Lee's in 1.5 seconds [38], and Commean et al.'s in 0.75 seconds [39]. Silhouetting and fringe projection differ in that silhouetting cannot pick up concavities of the residual limb while fringe projection can. However, for fringe projection, the subject with amputation must be positioned such that the residual limb is viewable from all directions, which might be difficult for some individuals. Commean et al. had their subject sit with the transtibial residual limb projected outward horizontally and the contralateral limb folded back under the chair [29]. Silhouetting can be accomplished with the subject in a normal standing posture, since $<290^{\circ}$ of viewing are needed to detect the entire residual limb surface. Testing showed repeatability error of 0.1 percent for silhouetting [38] and 0.6 percent for fringe projection [29].

Ultrasound. The residual limb is placed within a water-filled tank, and a noncontact ultrasound sensor is then moved around the inside of the tank to image the contour of the limb. A 3-D model of the limb is constructed, and residual limb volume is then determined [40-41]. Ultrasound confers the potential for imaging internal residual limb structures, thus offering additional insight not achieved with any of the preceding methods. However, ultrasonic imaging is very time consuming, with a scan time of $\sim 780$ seconds (13 minutes), making it highly susceptible to subject tremor and movement. Since initial efforts reported in 1996 [40] and 1997 [41], ultrasound has been used in only one other investigation in limb prosthetics [16].

Spiral X-Ray Computer Tomography. Spiral X-ray computer tomography (SXCT) uses X-ray images taken from multiple positions around the residual limb (computer tomography [CT] scan) to reconstruct both internal structures and the surface of the residuum [29,42-43]. Like ultrasound, it offers the investigator or practitioner additional insight beyond limb surface measurement, providing views of internal tissue structure and content. As reported in the mid-1990s [29,42-43], SXCT takes about 32 seconds to complete. Residual limb volume is calculated from a 3-D model assembled from the collected data. 
Smith et al. demonstrated residual limb volume errors of $<1.0$ percent for SXCT [42-43]. The measurement was susceptible to subject tremor and movement, but since the subject lay supine on a CT imaging table with the residual limb in the socket supported with foam blocks, these issues were not expected to introduce meaningful error. A challenge, though, was aligning images from sessions conducted on different days, since alignment was highly susceptible to slight position differences of the lead markers placed on the residual limb by the technician in different scanning sessions [43]. The lead markers were used as fiduciary points for alignment. Smith et al. later overcame this problem by using the bone as a reference [44]. These researchers also developed a sum projection, depth-shaded, cylindrical mapping technique to measure and present small changes in residual limb shape, using the displacement of lead markers to conduct this assessment [45].

Smith et al. described a two-part case study demonstrating use of SXCT for measuring residual limb shape [44]. The authors reported that intra- and intersession variability were similar $\left(13.6 \mathrm{~cm}^{3}\right.$ and $12.6 \mathrm{~cm}^{3}$, respectively) and that the differences between patients and between measurement sessions were significant, but that differences in scans taken within the same measurement session were not. In a single subject, differences in soft tissue volume, measured to be $18.0 \mathrm{~cm}^{3}$ for scans taken without the prosthesis donned, were within the range explained by random error, while differences in soft tissue volume for scans taken with different prostheses were $79.7 \mathrm{~cm}^{3}$ and outside the range explained by random error.

Laser Scanning. A plane or planes of laser light are projected onto the residual limb from multiple positions, and video images of the shapes the laser makes upon contact with the limb surface are recorded. Residual limb volume is calculated from a 3-D model constructed from the collected data [46,48-49].

Fernie et al. [46] and Oberg et al. [47] introduced laser scanning for residual limb volume measurement in prosthetics in the 1980s; however, performance results were not reported until 1995 [48]. The CAPOD laser scanner (CAPOD Systems AB; Karlskoga, Sweden) was cylindrical in shape with a central hole in which the residual limb was placed while the projector and scanner rotated about the inside of the cylinder. A strength of this design was that the plane of laser light was directed approximately perpendicular to the residual limb surface, which minimized distortion. A drawback of this design was that the subject needed to sit on a bicycle seat during scanning with minimal support, which is difficult for some individuals. Scanning took 10 seconds. Lilja and Oberg reported that measurements were within 2.5 percent of water displacement results and repeatability was 0.5 percent [48]. Three years later, Johansson and Oberg, using the same commercial scanner (CAPOD), reported comparable repeatability ( $0.4 \%)$, but volumes were much closer to water displacement measurements (0.3\%) [49]. It is unclear what modifications were made to the instrument to achieve this enhanced performance.

Magnetic Resonance Imaging. Commercial magnetic resonance imaging (MRI) equipment is used to image both internal structures and the surface of the residuum [50]. Residual limb volume is calculated using a 3-D model constructed from the collected data with commercial MRI system software. Imaging time with MRI is long, on the order of 592 seconds (approximately $10 \mathrm{~min}$ utes); thus the residual limb must be well-stabilized during scanning. Buis et al. [50], the only investigators to report using MRI for residual limb volume assessment, claimed that MRI could be used to establish a consistent bony structure grid, which could then be used as a reference for residual limb volume comparisons, similar to Smith et al.'s [44] technique. However, Buis et al. did not report volume data [50].

Bioimpedance. Bioimpedance measures the resistance of the residual limb to electrical current, which is related to ECF and intracellular fluid volumes [53]. Current injecting and voltage sensing electrode pairs are placed on the residual limb. Current is applied across a range of frequencies, and the electrical impedance is measured $[7,51]$. The bioimpedance data are converted to residual limb fluid volume using computational models described in the literature [54]. Bioimpedance is effective for measurement of residual limb fluid volume change in conductive tissues within the limb, predominantly muscle and skin [55], which are expected to be the primary tissues that change volume in persons with lower-limb amputation [6]. It is important to note that bioimpedance reflects residual limb fluid volume, not residual limb volume like the other techniques described previously. Bioimpedance analysis has been shown to be highly correlated $(r>0.9)$ with ECF volume as measured with MRI in nondisabled limb segments [56] and with bromide dilution techniques in whole-body analysis [57]. The good reproducibility on calves of nonamputated limbs led Zhu et al. to suggest that 
bioimpedance analysis is sufficiently accurate and sensitive to effectively monitor the degree of hydration during dialysis treatment [58]. Sanders et al. applied bioimpedance analysis to the residual limbs of persons with transtibial amputation using a commercial instrument modified for this purpose and were able to resolve limb fluid volume changes during standing and before-to-after bouts of walking [51]. Repeatability during standing on nondisabled subjects was reported as 0.2 percent.

Measurement Techniques Summary. Though these numerous techniques have been developed to measure residual limb volume change, only some have been used in clinical studies on people with lower-limb amputation, including water displacement, casting + water displacement, anthropometric measurements, optical scanning, ultrasound, MRI, laser scanning, and bioimpedance. Results from those studies are described in the sections on group II and III publications. Methods developed but not used in clinical studies to date to assess limb volume include contact probes and SXCT.

\section{Sources of Error and Characteristics of Measurement Techniques}

A number of challenges make measurement of residual limb volume difficult. Each technique listed previously has its advantages and disadvantages. Here, these challenges are described and the relevance to the different measurement techniques discussed.

Distortion of Limb Shape. Measurement methods that contact the residual limb when the prosthesis is doffed distort its shape and affect the residual limb volume measurement of interest. Of the techniques listed previously, anthropometric measurements, contact probes, and casting + water displacement are the most susceptible to this error since they apply the most force to the limb. Skin compresses easily under very little stress [59]. Vannah and Childress reported that lower-limb soft tissues compressed $3 \mathrm{~mm}$ under just $4 \mathrm{kPa}$ (0.6 psi) of applied pressure [60]. For an average-size residuum and using a well-accepted model in the literature $(20 \mathrm{~cm}$-long cylinder with $5 \mathrm{~cm}$ radius half-sphere on the distal end [14]), a researcher or clinician pressing a probe $1 \mathrm{~mm}$ into the limb over its entire surface will induce a 4 percent reduction in residual limb volume. Thus, if small volume changes are of clinical interest, such as those occurring over the course of a day (reported as between -1.5 and +2.0 percent on a series of eight subjects with transtibial amputation [12]), then contact measurement methods are not appropriate. However, to measure postoperative residual limb volume changes over several months (approximately 17\%-35\% [11]), contact measurements are acceptable.

Postdoffing Volume Change. Part of the challenge with all of the measurement techniques listed here, with the exception of SXCT and bioimpedance, is that they measure volume of the residual limb while it is outside of the prosthetic socket. The challenge is that residual limbs change volume over time after doffing, and these changes might be substantial. For example, Zachariah et al., using a silhouetting optical method, reported residual limb volume changes on six transtibial subjects of between 0.2 and 17.0 percent within the first 5 minutes after doffing [6]. Further, the degree of postdoffing residual limb volume change varied considerably from subject to subject, and the rate of residual limb volume change varied between sessions for the same subject. Because so few subjects were tested, dependence of postdoffing volume changes and session-to-session volume changes on subject characteristics could not be investigated. In the future, investigation of a large subject population might reveal these relationships. Thus, clinical investigations intended to assess residual limb volume changes for different treatments or socket designs during ambulation are more reliable if measurements are made while the prosthesis is donned. If postdoffing methods must be used, then the postdoffing volume comparisons should be made within each subject as opposed to between subjects and the time between doffing and volume measurement should be consistent from trial to trial. Methods that measure volume quickly after doffing are preferred since the residual limb volume change will be less. Of the techniques listed previously, optical methods, bioimpedance, and anthropometric measurements are the fastest; contact probes, water displacement, casting + water displacement, SXCT, laser scanning, and MRI are slower (with the exception of one laser scanner system that was reported to be very quick [46] but was never used for clinical investigation).

Subject Movement. If the scan time of the measurement technique is long and the subject moves during scanning, the measurement will be affected, reducing accuracy of the volume measurement. This problem has been recognized by numerous researchers [28-29,32,37$41,43-44,46,50]$. The challenge is that a relatively small subject movement can induce a large volume error. Fernie and Holliday, for example, had an attrition rate of 35 percent because measurements could not be taken reliably 
with their water displacement technique on many subjects (17 of 49 persons with lower-limb amputation) [14]. Some devices are designed to correct for subject movement. For example, Smith et al. [44] and Buis et al. [50] used the bone as a reference shape to align data collected over the scanning period. In a different study, tracking of reflective markers affixed to the limb surface was used to correct limb movement error for an optical silhouette scanner (1.5-second image time), reducing error 40 percent (from $1.0 \%$ down to $0.6 \%$ ) [38]. Tracking of markers placed on the limb surface performed best if the markers were well distributed in the imaging field. Certainly, technologies with short scan times are less susceptible to subject movement problems. Thus, for subjects who have difficulty sitting still, quick optical methods, bioimpedance, and anthropometric measurements are preferred over contact probes, water displacement, casting + water displacement, and laser scanning. Similar to a camera with a quick shutter speed, these quicker measurement techniques will be affected with less distortion.

Accuracy and Resolution. Accuracy and resolution of the measurement instrument are important to the quality of the data. Some published studies report instrument error (Table 1). These assessments were limited by the capabilities of gold standard test systems at the time the instruments were developed. In most cases, no gold standard more accurate than the instrument under development was available. Because of this situation and because it was of interest to assess volume change as opposed to volume, several investigators reported the repeatability performance of their instrument. Good repeatability demonstrates that random error from measurement to measurement, an important issue when small changes in volume are of interest, is minimal. In Table 1, we have listed errors for each study most relevant to volume change measurement since it is the change in volume, as opposed to absolute volume, that is of interest. Errors were either stated directly in the articles or calculated by us from results presented. In some cases, only the volume accuracy relative to a gold standard was available. Table 1 also lists the advantages and disadvantages of each technique. When investigators reported results from multiple limb models or subjects with amputation, the mean error is listed in the "Volume Change Error" column. Results from evaluations using simple cylindrical model shapes are not reported in this column because we do not believe they are relevant to clinical application. Unless specifically stated, the errors listed in this column for studies on residual limb replicas do not include errors present in human subject testing (contact, scan time, patient movement). Thus, instruments sensitive to the limitations listed in Table $\mathbf{1}$ and for which volume errors were measured using limb replicas will likely have greater errors when used on persons with amputation.

Some scanner products developed to capture limb shape for prosthetic socket design purposes have the potential for use in clinical investigations of residual limb volume change after doffing. However, to determine whether they are useful for this purpose, we must test systems against pairs of models of known volume difference. Results from such tests should then be included in product information to inform the practitioner and researcher of the capabilities and applications of the scanner.

It is worth noting that for devices that scan limb shape (e.g., contact probes, optical scanners, ultrasound, SXCT, and laser scanners) spatial resolution (typically reported in millimeters) and volume resolution (typically reported in percent) are different. Volume error is more forgiving. If a scanner measuring limb shape demonstrates a randomly and normally distributed radial error about zero, then its mean radial error is very low, which is good for volume measurement, because its volume resolution, which is proportional to the sum of the radial measurement data, is thus also low. So even though a scanner might have mediocre spatial resolution, if its radial error is randomly and normally distributed about zero, then it might still be a good instrument for volume measurement. It would be helpful if product information for scanners reported performance of the device for volume measurement not just spatial resolution. It is essential to conduct these assessments on residual limb model shapes of known volume, as opposed to simple circular cross-section shapes. Laser scanner errors, for example, are typically higher if the angle of incidence of the plane of laser light with the residual limb surface is shallow. A very accurate laser scanner that rotates physical models so that the plane of laser light is approximately perpendicular to the test object (e.g., NextEngine Inc; Santa Monica, California) could be useful in setting a gold standard for evaluation of scanning systems for postdoffing residual limb volume assessment. 


\section{Comparing Volumes from Different Scans}

A challenge for researchers investigating the effects of different socket designs, rehabilitation treatments, or other clinical variables on residual limb volume change is that typically residual limb volumes from different trials need to be compared. This effort requires aligning the different residual limb shapes, which is not a trivial issue. Comparison of socket shapes, a related challenge that has been addressed in commercially available socket-design software, is simpler because typically distinct anatomically linked features (e.g., patellar tendon bar; relief for the tibial tubercle or fibular head) facilitate proper alignment. Limb shapes, however, often lack such distinct contours at these locations. When markers are placed on the residual limb and are not moved between scans, the issue is of no concern because the alignment algorithm simply lines up the markers when processing the scans [45]. However, for longer durations between sessions, such as weeks or months, during which markers are removed or rub off, the analysis is more difficult. Consistent positioning of markers from one session to another is difficult and introduces much error to the analysis [43]. For modalities such as SXCT, MRI, and ultrasound that image internal limb structures, the bone can be used as a reference since its shape changes much less than that of soft tissues. However, technologies available for use in the prosthetist's clinic do not typically image internal structures. Instead, shapes need to be aligned using the shapes of the residual limb surface.

There is a rich history of increasing sophistication in techniques for aligning socket or limb shapes. These are summarized in Table 3. Sidles et al. minimized volume difference to align socket and limb shapes for $\mathrm{CAD} /$ CAM socket design [61], a procedure eventually incorporated into ShapeMaker, one of the early prosthetic design software packages. Two subsequent groups, Chahande et al. and Jimenez et al., used anatomical landmarks for alignment of residual limbs [62-63]. Lemaire and Johnson implemented the alignment of top and bottom slice centroids to align socket shapes [64]. Smith et al. used a least squares computational optimization procedure to align solid models constructed from SXCT scans [44]. Zachariah et al. found that while minimizing volume differences resulted in well-aligned residual limbs that had localized shape differences, the technique was not effective for aligning residual limbs with global shape differences [65]. Thus, they developed and evaluated a new method for aligning residual limb shapes using a combination of the mean absolute radii difference and alignment of surface normals. The surface normals, lines extending perpendicularly outward from the limb surface, were very sensitive to slight contour differences

Table 3.

Summary of residual limb alignment techniques.

\begin{tabular}{lll}
\hline \multicolumn{1}{c}{ Technique } & \multicolumn{1}{c}{ Study } & \multicolumn{1}{c}{ Application Shapes } \\
\hline Minimization of Volume Differences & Sidles et al. [1] & Residual limbs, sockets \\
Anatomical Landmarks & Smith et al. [2] limbs, using markers \\
& Chahande et al. [3] & Residual limbs \\
Top and Bottom Slice Centroids & Jimenez et al. [4] & Residual limbs \\
Minimization of Volume Differences and & Lemaire \& Johnson [5] & Sockets \\
Residual limbs
\end{tabular}

Surface Normals

1. Sidles JA, Boone DA, Harlan JS, Burgess EM. Rectification maps: A new method for describing residual limb and socket shapes. J Prosthet Orthot. 1989; 1(3):149-53. DOI:10.1097/00008526-198904000-00009

2. Smith KE, Commean PK, Vannier MW. Residual-limb shape change: Three-dimensional CT scan measurement and depiction in vivo. Radiology. 1996; 200(3):843-50. [PMID: 8756942]

3. Chahande A, Billakanti S, Walsh N. Lower limb shape characterization using feature extraction techniques (noncontact laser scanning). Proceedings of the 16th Annual International Conference of the IEEE Engineering in Medicine and Biology Society; 1994 Nov 3-6; Baltimore, MD. Los Alamitos (CA): IEEE; 1994. p. $482-83$.

4. Jimenez D, Darm T, Rogers B, Walsh N. Locating anatomical landmarks for prosthetic design using ensemble neural networks. Proceedings of the International Conference on Neural Networks; 1997; Houston, TX. Piscataway (NJ): IEEE; 1997. p. 81-87. Available from: http://ieeexplore.ieee.org/xpl/freeabs_all.jsp?arnumber $=611641$.

5. Lemaire ED, Johnson F. A quantitative method for comparing and evaluating manual prosthetic socket modifications. IEEE Trans Rehabil Eng. 1996;4(4):303-9. [PMID: 8973956] DOI:10.1109/86.547931

6. Zachariah SG, Sorenson E, Sanders JE. A method for aligning trans-tibial residual limb shapes so as to identify regions of shape change. IEEE Trans Neural Sys Rehabil Eng. 2005;13(4):551-57. [PMID: 16425837] DOI:10.1109/TNSRE.2005.858459 
and thus were a more sensitive measure for shape error than were absolute radii differences. Results showed that this algorithm was effective for proper alignment of residual limbs with both regional and distributed shape differences. Potentially, this algorithm can be extended into commercial software for the alignment of residual limb shapes.

From the success of CAD/CAM software packages in the prosthetic industry, it is reasonable to suspect that existing algorithms to align and compare socket shapes are adequate for clinical purposes. However, other than Zachariah et al.'s assessment, no studies have reported performance of residual limb shape-alignment algorithms [65]. If commercially available scanning systems are to be used to measure and compare residual limb volumes from one time to another, assessment of shape-alignment algorithms will be needed.

\section{Availability}

Instruments readily available to the researcher and prosthetist for residual limb volume measurement include standard and spring-loaded tape measures and calipers, contact probes, water displacement (using a scale to measure mass change of the water), casting + water displacement, optical scanning, and laser scanning. SXCT and MRI are available at some clinics and hospitals, but no commercially available system designed specifically for use in prosthetics exists.

With regards to measurement of residual limb volume, some techniques have not yet transitioned from the research arena to clinical practice. In particular, these include ultrasound, SXCT, and bioimpedance. The long scanning time and water submersion required for ultrasound make it unattractive for further development in residual limb volume measurement compared with other modalities. SXCT produced very good results [66] and could become a valuable tool in the future, provided prosthetic SXCT algorithms are incorporated into equipment available to researchers and prosthetists. Bioimpedance has just begun to be used for prosthetic applications $[7,51]$. The technology has good potential for clinical use because it can be implemented by practitioners in their offices at relatively low expense. However, it will take time to develop appropriate instruments, clinical protocols, models, and analysis algorithms to interpret the data from this new modality.
Sources of Error and Characteristics of Measurement Techniques Summary

Trade-offs in Measurement Techniques. When choosing a technique for measurement, the user needs to consider what qualities are most relevant to his or her application and then choose wisely. For example, if one were interested in measuring postoperative limb volume changes, circumferential measurements with a springloaded tape measure used with a cylindrical or frustrum model would likely be sufficient. If one were interested in comparing residual limb volume after using one socket design versus another socket design on a patient, insocket tests would be best (SXCT, bioimpedance).

Measurement Technique Can Affect Study Results. When reading clinical research reports, the reader needs to consider the results in light of the method used. We need to ascertain whether error from the measurement technique was greater than the residual limb volume changes reported for the variable of interest in the study. Investigators should conduct assessments of their instruments and report instrument errors to help the reader interpret the results.

Products Developed for Capturing Limb Shape Might Be Viable for Measurement of Residual Limb Volume Change. Manufacturers of scanners need to evaluate their products on pairs of physical limb models with known volume differences to determine their capabilities to assess residual limb volume change. Techniques to correct for limb movement during scanning are essential if the scan time is longer than $\sim 1$ second [38]. Further, to compare limb shapes, manufacturers need to incorporate shape-alignment algorithms reported in the literature or extensions from them into commercial products and then potentially enhance them through clinical use. The effects of postdoffing time on scanner measurements need to be considered when interpreting data so that we know for which clinical applications postdoffing residual limb volume data are useful and for which they are not.

\section{Group II: How Does Residual Limb Volume Change Affect Clinical Outcomes?}

Of the 13 studies included for review in group II [614,16,67-69], some studies used anthropometric measurements to estimate time to fitting of a prosthesis [8$11,67]$ without necessarily reporting the actual circumference data [9-10,67]. This interval (time to fitting of a 
prosthetic limb) was proposed as a pseudomeasure for when the limb was no longer edematous.

Techniques used in clinical studies to assess residual limb volume and shape included anthropometry [810,67-68], water displacement $[8,14]$, noncontact scanners (optical and laser) [6,11-13], bioimpedance [7], MRI [69], and ultrasound [16]. All studies focused exclusively on persons with transtibial amputation with the exception of Singh et al. [16], Wong and Edelstein [10], and Fernie and Holliday [14].

The reviewed articles included four experimental studies (two randomized control trials [10,67] and two controlled trials [8-9]) and nine observational studies (two cohort studies [14,16], six case series [6-7,11$13,68]$, and one case study [69]).

Studies were divided into those that assessed early postamputation residual limb volume change [8-11,1314,16,67-69] and those that assessed residual limb volume change in mature residual limbs $[6-7,12,14]$.

\section{Early Postamputation Phase}

Characterizing Residual Limb Volume Change in Early Postamputation Phase. Five studies [8,11,14,6869] were identified that attempted to characterize residual limb volume in the early postamputation phase, and one study described discrete fluid collections almost immediately postamputation [16].

Stabilization of Postamputation Edema. Using different measurement techniques (anthropometric measurements [8,68], laser scanner [11], and water displacement $[8,14])$, four research groups attempted to identify the time postamputation when residual limb volume had stabilized sufficiently for prosthetic fitting to be attempted.

Persson and Liedberg assessed residual limb volume in 93 transtibial amputations during the first 12 weeks postamputation [68]. They used anthropometric measurements taken at 2, 3, 4, 6, and 12 weeks to calculate residual limb volume. While residual limb dimensions were described, amputee characteristics such as cause of amputation and age were not, making it difficult to know to whom the results might best apply. Residual limb volume decreased by $7.3 \pm 10.6$ percent over 12 weeks. This change was likely outside of the measurement error (Appendix 6, available online only).

Lilja and Oberg assessed residual limb volume in 11 subjects with transtibial amputation due to PVD during the first 23 weeks (161 days) postamputation [11]. They used a noncontact laser system (CAPOD) to measure volume once a week for the first 8 weeks, every second week for the next 6 weeks, and once a month for the remainder of the study. Subjects were managed postoperatively according to standard practices that involved elastic bandaging. Data were normalized relative to the 5th and 160th days postamputation, and these data were calculated via interpolation if actual measurements were not available for those days. The authors reported that a rapid decrease in residual limb volume occurred in the early postoperative phase, with reduction slowing over time. The data were found to fit a negative exponential function with a mean coefficient of determination of 0.90 (range 0.81 to 0.96 ). Lilja and Oberg hypothesized that the initial rapid reduction in residual limb volume was due to resolution of postoperative edema and that the later, slower reduction was due to muscle atrophy [11]. Total range of volume decrease over 160 days was 17 to 35 percent. They calculated that for most of their subjects, residual limb volume stabilized to within 5 percent by 120 days postamputation. The volume changes reported $(>5 \%)$ are outside of the measurement error (Appendix 6, available online only).

Fernie and Holliday reported data for 32 persons with lower-limb amputation: 18 new amputees (all but one were transtibial) were followed for 2 years (600 days or 104 weeks) and 14 subjects with mature residual limbs (7 transfemoral, 1 knee disarticulation, and 6 transtibial) were followed for 1 year or more (>352 days) [14]. These researchers measured residual limb volume change over time using water displacement and assessed differences between groups. Unfortunately, substantial issues with reliability due to subject movement within the test measurement led to 35 percent attrition in the available data (49 subjects were initially recruited but data were reported for only 32). Thus, the data reflected only people with amputation who could keep their residual limbs still during the measurement protocol. The makeup of these subjects in terms of health status and demographics was not reported. Fernie and Holliday reported that new amputees experienced decreases in limb volume over the first 100 to 200 days by 5 percent and by 10 percent over 200-600 days [14]. These reported volume changes were likely outside of the measurement error (Appendix 6, available online only). Beyond 200 days, the authors were unable to identify a consistent pattern of residual limb volume change, noting that postoperative residual limb shrinkage interacted with increasing body weight. 
All subjects in this study increased body weight by 2 percent in 1 year, up to 30 percent over 2 years. By comparison, Lilja and Oberg reported that body weight increased by approximately 3.4 percent during 23 weeks postoperative follow-up in 11 subjects with amputation due to PVD [11]. Fernie and Holliday suggested that difficulty with donning the prosthetic socket occurred when the residual limb volume increased by 3 to 5 percent [14].

Golbranson et al. conducted a controlled trial of three different methods to stabilize residual limb volume on 36 geriatric individuals with vascular disease and unilateral transtibial amputation [8]. Residual limb volume was calculated using both water displacement and circumference measurements. Measurements were made once a week during the "initial limb maturation phase" and bimonthly after the fitting of the first permanent prosthesis. Although their linear regression would suggest an 11 percent decrease in residual limb volume over the first 100 days postamputation, the authors reported that volume stabilization was impossible to determine in more than half their subjects. Golbranson et al. estimated that the accuracy of their volume measurements was about $30 \mathrm{~mL}$ because of three sources of error: variation in water temperature and density, motion-induced wave action caused by the subject, and muscle contraction within the residual limb [8]. Other issues may also have affected their results (Appendix 6, available online only). Thus the lack of a consistent volume change pattern might have been a result, at least in part, of limitations in the measurement technique.

All four studies characterizing residual limb volume change during the early postoperative phase $[8,11,14,68]$ suggest that transtibial residual limb volume reduction occurs over at least the first 100 days (14 weeks) postamputation. The authors of two of these studies state that in persons with transtibial amputation followed for 12 to 14 weeks, generalized edema decreases with reduction slowing over time $[11,68]$. The results from one study that followed subjects for up to 23 weeks suggest that no consistent pattern of residual limb volume change in persons with transtibial amputation beyond 100 to 200 days postamputation exists [14].

\section{Effect of Muscle Atrophy on Residual Limb Volume.}

We reviewed one case series of three persons with transtibial amputation (1 bilateral, 2 unilateral) that assessed the effect of muscle atrophy on cross-sectional area of the residual limb during the early postoperative period [69]. MRI was used at 2, 6, and 28 weeks after amputation to obtain transverse images and calculate circumferences and cross-sectional area at 33, 50, and 66 percent of the residual limb length. The total cross-sectional area and circumference decreased very quickly between the first and second examination in all subjects and increased slightly in all cases between the second and third examination. The authors noted that the amount of subcutaneous fat present in the residual limb increased over the study period. The cross-sectional area of all muscles decreased between the first and second examination, but between the second and third examination, the crosssectional area decreased for the medial head of the gastrocnemius, soleus, and triceps surae but increased for the lateral head of the gastrocnemius and the anterior tibial muscles. The authors hypothesized that the increase in cross-sectional area may be related to the health of the subject at the time of amputation (elderly, PVD, poor condition), where initially a catabolic phase caused muscle breakdown and later an anabolic phase built up muscle tissue. The reported changes were outside the measurement error (Appendix 6, available online only).

Presence and Effect of Discrete Fluid Collections. A single cohort of 105 lower-limb amputees (41\% transfemoral and 59\% transtibial) was assessed during the postoperative phase to evaluate the incidence and consequence of discrete fluid collection in the residual limb (as opposed to generalized edema) [16]. The residual limb was scanned on admission to the rehabilitation unit (median 19 days postsurgery, range 9 to 62 days) using a handheld ultrasound scanner that automatically calculated the volume of a bolus of fluid within the residual limb. Twenty-eight limbs (27\%) had significant fluid collections, with a median volume of $38.5 \mathrm{~mL}$ (range 16 to $216 \mathrm{~mL}$ ). Fluid collections were rescanned every 2 to 3 days to monitor progress. Discrete fluid collections were more common in persons with transfemoral amputation than transtibial. All collections diminished on subsequent scanning, with 81 percent undetectable at 10 days. The authors reported that no significant difference was found in age, sex, comorbidities, proportion achieving limb fitting, or psychological symptoms as a result of these discrete fluid collections. However, individuals with fluid collection took 9.5 days longer to be ready to be fit with a prosthesis and had a 10-day longer inpatient stay. While the reported changes were likely outside of the measurement error (Appendix 6, available online only), the determination of readiness for prosthetic fitting was rather 
vague, making this the most distinct limitation of the study.

Estimating Time to First Prosthetic Fitting. Time to fitting of the first prosthetic limb in persons with transtibial amputation was assessed in seven studies we reviewed [8-11,14,67-68] (Table 4).

Effect of Early Ambulation on Time to Fitting of First Prosthesis. Two studies evaluated the effect of early walking with a temporary prosthesis on residual limb volume [8,67]. Golbranson et al. [8] conducted a controlled trial of three different methods to stabilize residual limb volume (elastic wrap, plaster cast + pylon, and plastic laminate socket + pylon) in 36 geriatric subjects. The authors reported the mean \pm SD and standard error of the mean
(SEM) for "volume over the study" for each group as elastic wrap $997 \pm 312 \mathrm{~mL}$ (SEM = 87), plaster cast $908 \pm 212$ $\mathrm{mL}(\mathrm{SEM}=67)$, and plastic laminate socket $843 \pm 368$ $\mathrm{mL}(\mathrm{SEM}=102)$. They also report that average volume decreased significantly in the ambulatory groups but not in the nonambulatory elastic wrap group; average circumferences decreased significantly in the ambulatory groups but increased significantly in the nonambulatory elastic wrap group. Although the changes measured were expected to be outside of the measurement error (Appendix 6, available online only), the comparator conditions were confounded with regards to their ability to attribute changes in residual limb volume to early ambulation since both socket type and pylon use changed between conditions.

Table 4.

Summary of residual limb volume change in early postamputation phase and time to first fitting of prosthesis.

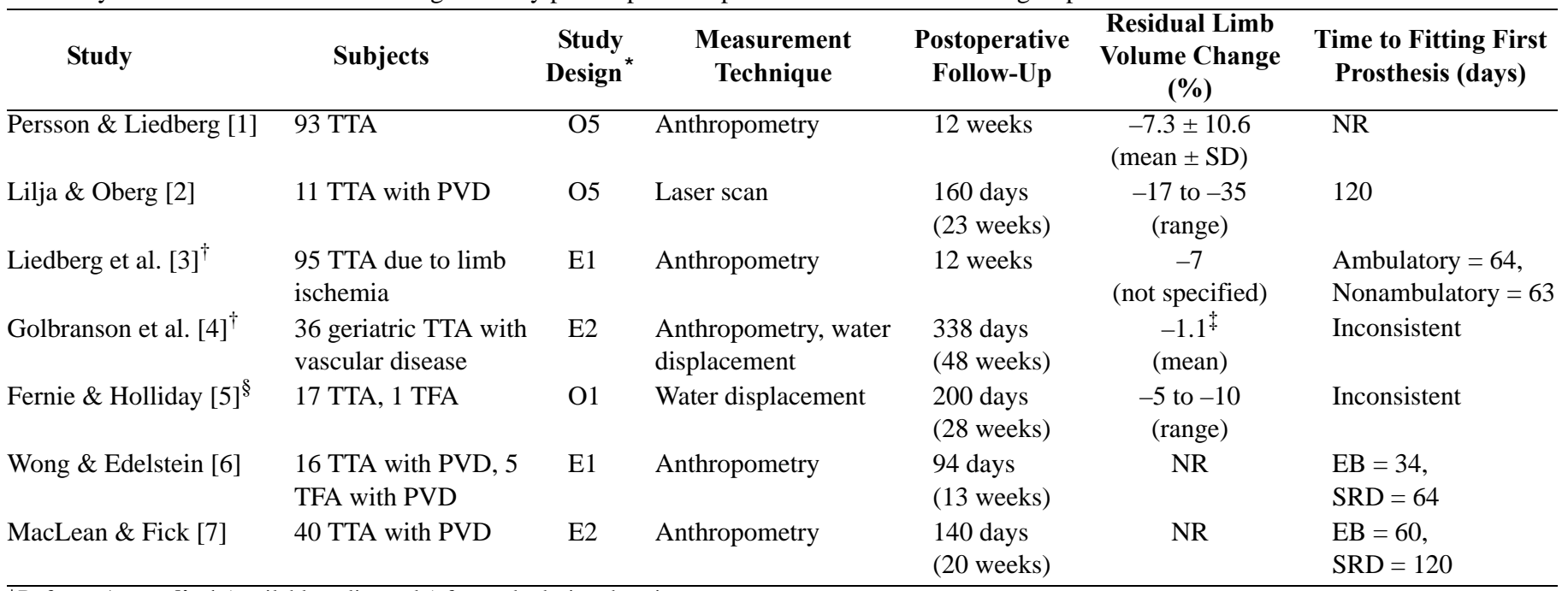

${ }^{*}$ Refer to Appendix 1 (available online only) for study design descriptors.

${ }^{\dagger}$ Although these studies compared multiple groups, total residual limb volume change data were not different across groups and so data for entire study population were included.

${ }^{\ddagger}$ Residual limb volume change estimated from slope of regression curve for entire group, which was $0.011 \%$ per day over 100 days.

${ }^{\S}$ Data for new residual limbs only.

1. Persson BM, Liedberg E. A clinical standard of stump measurement and classification in lower limb amputees. Prosthet Orthot Int. 1983;7(1):17-24. [PMID: 6856447]

2. Lilja M, Oberg T. Proper time for definitive transtibial prosthetic fitting. J Prosthet Orthot. 1997;9(2):90-95. DOI:10.1097/00008526-199700920-00009

3. Liedberg E, Hommerberg H, Persson BM. Tolerance of early walking with total contact among below-knee amputees—A randomized test. Prosthet Orthot Int. 1983;7(2):91-95. [PMID: 6622240]

4. Golbranson FL, Wirta RW, Kuncir EJ, Lieber RL, Oishi C. Volume changes occurring in postoperative below-knee residual limbs. J Rehabil Res Dev. 1988;25(2):11-18. [PMID: 3361456]

5. Fernie GR, Holliday PJ. Volume fluctuations in the residual limbs of lower limb amputees. Arch Phys Med Rehabil. 1982;63(4):162-65. [PMID: 7082139]

6. Wong CK, Edelstein JE. Unna and elastic postoperative dressings: Comparison of their effects on function of adults with amputation and vascular disease. Arch Phys Med Rehabil. 2000;81(9):1191-98. [PMID: 10987161] DOI:10.1053/apmr.2000.3780

7. MacLean N, Fick GH. The effect of semirigid dressings on below-knee amputations. Phys Ther. 1994;74(7):668-73. [PMID: 8016199$]$

$\mathrm{EB}=$ elastic bandage, $\mathrm{NR}=$ not reported, $\mathrm{PVD}=$ peripheral vascular disease, $\mathrm{SD}=$ standard deviation, $\mathrm{SRD}=$ semirigid dressing, $\mathrm{TFA}=$ transfemoral amputation, TTA $=$ transtibial amputation. 
Liedberg et al. conducted a randomized controlled trial of the temporary "tulip" limb in 95 persons with transtibial amputation due to limb ischemia, comparing it with existing therapy without a temporary limb, which was provided to both groups [67]. Residual limb volumes were calculated using anthropometric measurements of the residual limb and a model shaped as an arbitrary cut cone [68]. Measurements were made at 2, 4, 6, and 12 weeks. The authors do not provide volume data; thus, the reliability of the measurement cannot be assessed. They report that "there was a decrease in volume of about 7 percent from the first measurement at 2 weeks to the third measurement at 12 weeks, but there was no difference between groups" [67]. Methods used to assess this difference were not described.

Estimating Volume of Stockings/Socks. Fernie and Holliday used a cylinder with one hemispherical end, a radius of $5 \mathrm{~cm}$, and a total length of $25 \mathrm{~cm}$ to approximate a typical prosthetic socket and calculated the volume reduction caused by the addition of two five-ply socks (the amount considered clinically acceptable) as 10 percent [14]. However, their records suggested that difficulty with donning of the prosthetic socket occurred when the residual limb volume increased by 3 to 5 per- cent. Lilja and Oberg and Lilja et al. reported that the volume of one five-ply stocking was 5.2 percent and two five-ply stockings was 9.4 percent of the residual limb volume for a limb model of $1192.1 \mathrm{~mL}$ in volume $[11,13]$.

\section{Mature Residual Limbs}

Characterizing Residual Limb Volume and Shape in Mature Residual Limbs. Our review identified two studies that provided data regarding short-term (within a day) residual limb volume change in mature residual limbs [6-7] and two studies that explored longer term (2 weeks) residual limb volume change in mature residual limbs [12,14]. Results are summarized in Table 5.

\section{Short-Term Changes in Residual Limb Volume.}

While two studies provide data that begin to characterize short-term changes in residual limb volume, their main objectives were to demonstrate utility of the techniques used, a custom optical scanner [6] and bioimpedance analysis [7], and to gain insight into how quickly fluid moves within the residual limb. It is worth noting that these two techniques measure slightly different aspects of residual limb volume, with bioimpedance measuring

Table 5.

Summary of short- and long-term residual limb volume changes.

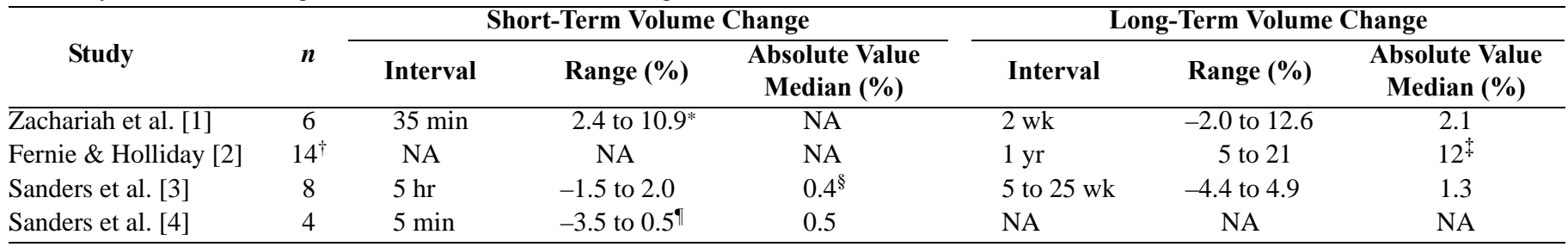

${ }^{*}$ Measurement made right after walking and doffing.

${ }^{\dagger}$ Clarified based on Fernie et al. report [5].

${ }^{\ddagger}$ Mean volume change reported.

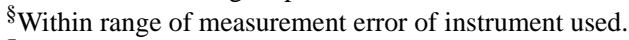

"Measurements made during walking.

1. Zachariah SC, Saxena R, Fergason JR, Sanders JE. Shape and volume change in the transtibial residuum over the short term: Preliminary investigation of six subjects. J Rehabil Res Dev. 2004;41(5):683-94. [PMID: 15558398] DOI:10.1682/JRRD.2003.10.0153

2. Fernie GR, Holliday PJ. Volume fluctuations in the residual limbs of lower limb amputees. Arch Phys Med Rehabil. 1982;63(4):162-65. [PMID: 7082139]

3. Sanders JE, Zachariah SG, Jacobsen AK, Fergason JR. Changes in interface pressures and shear stresses over time on trans-tibial amputee subjects ambulating with prosthetic limbs: Comparison of diurnal and six-month differences. J Biomech. 2005;38(8):1566-73. [PMID: 15958212] DOI:10.1016/j.jbiomech.2004.08.008

4. Sanders JE, Harrison DS, Allyn KJ, Myers TR. Clinical utility of in-socket residual limb volume change measurement: Case study results. Prosthet Orthot Int. 2009;33(4):378-90. [PMID: 19961297] DOI:10.3109/03093640903214067

5. Fernie G, Holliday P, Logg R. An investigation of oedema and shrinkage in the stumps of lower limb amputees [Internet]. Health and Welfare Canada Final Report on Project \# 606-1317-41, West Park Research, West Park Hospital, Toronto, Ontario. Toronto Ontario. 1980. Available from: http://www.amputee-coalition.org/inmotion/mar apr 06/prosthetic socks.html.

NA = not applicable. 
residual limb fluid volume.

Zachariah et al. described a case series quantifying short-term changes in residual limb volume that occur after a short period of walking and immediately postdoffing of the prosthetic socket on two occasions 2 weeks apart [6]. The residual limb shape and volume of six persons with unilateral transtibial amputation, five with limb amputation as a result of traumatic injury, and one from meningitis were assessed using an optical scanner and silhouetting technique. The authors reported that mean volume increase ranged from 2.4 to 10.9 percent (median $6.0 \pm 3.6 \%$ ), with the rate of increase highest immediately upon socket removal and decreasing over the rest of the 35-minute test period. Most of the volume increase occurred in the first 8 minutes. No consistent differences were found in proximal to distal circumference changes. Volume differences 2 weeks apart ranged from -2.0 to +12.6 percent (absolute value median 2.1\%) and were less than those observed within a 35-minute session. Changes measured were outside of the measurement error (Appendix 6, available online only).

Sanders et al. described a case series quantifying short-term changes in residual limb fluid volume during walking in four persons with transtibial amputation, two with and two without comorbidities [7]. Limb positioning and weight-bearing levels during testing were standardized. For all subjects, residual limb ECF volume decreased during standing, but during subsequent walking, the pattern of ECF volume seemed to depend on the health of the subject. The limb of subjects without comorbidities increased in ECF volume $(0.2 \%$ to $0.9 \%$ of ECF volume at the outset of the trial), while that of those with comorbidities decreased $(-0.2 \%$ to $-3.5 \%$ of ECF volume at the outset of the trial). Based on data presented for all subjects, the median absolute fluid volume change during walking was 0.5 percent of the ECF volume at the outset of the trial. The reported changes were outside the measurement error (Appendix 6, available online only). While compression from the socket occurred during walking, Sanders et al. hypothesized that in subjects without comorbidities, increased cardiovascular activity was a stronger influence when walking was initiated, driving fluid out of the limb [7]. The authors further indicated that presenting subjects with volume data was effective at improving compliance with recommended care.

\section{Long-Term Changes in Residual Limb Volume.}

Two quite different case series [12,14] reported on longterm residual limb volume in mature residual limbs.
Using a water displacement method, Fernie and Holliday followed 14 subjects with mature residual limbs for up to 1 year, with 7 subjects followed for 1 additional year [14]. Subject numbers were unclear in the article but were confirmed in the final report of the project written for the funding agency [70], which was obtained directly from Fernie. Results were reported only for subjects who were capable of standing still during measurement. Mean volume change was 12 percent (range 5\%-21\%), and residual limb volume fluctuations were reported to correspond to body weight.

Sanders et al. followed 8 persons with unilateral transtibial amputation as a result of traumatic injury at 5 week intervals for 25 weeks (180 days) [12]. They assessed interface pressures, shear stresses, and residual limb shape. Changes in interface stress and residual limb volume and variability in the change in residual limb cross-sectional area were compared for diurnal and longterm intervals. A custom optical scanner similar to that used by Zachariah et al. [6] was used to assess residual limb shape. The authors stated that absolute interface pressure and resultant shear stress changes for all sessions conducted 10 to 25 weeks apart were significantly greater than diurnal changes, except interface pressures at 10 weeks. The authors also observed that residual limb cross-sectional area changes for diurnal intervals were relatively uniform down the length of the residual limb, but for 25 week intervals, the changes were more localized and had a significantly larger variance. Diurnal residual limb volume change ranged from -1.5 to +2.0 percent. Median absolute diurnal volume change, calculated as 0.4 percent from the results presented, was within the measurement error $(0.1 \%-1.0 \%)$. Long-term changes (5-25 weeks apart) ranged from -4.4 to +4.9 percent, with a median absolute change of 1.3 percent, which was outside of the range of measurement error (Appendix 6, available online only). One issue that arose with this study was the inability to follow subjects for longer than 25 weeks because of socket fit problems when limb volume reductions of more than $\sim 4$ percent or limb volume increases of more than $\sim 5$ percent occurred. The study protocol did not allow socket modifications.

Effect of Muscle Activity and Liners on Mature Residual Limb Volume. Lilja et al. reported on a case series of 16 persons with transtibial amputation (mean age 72 , range 57 to 83 ) that assessed the effect of muscle cocontraction and silicone liners on residual limb volume and the implications for casting and socket fit [13]. The 
CAPOD noncontact laser scanning system was used to assess residual limb volume between contracted and relaxed limbs with and without a liner. Lilja et al. reported that post hoc power for volume change with and without the silicone liner was 0.82 . The authors also reported that a significant increase in volume of $5.8 \pm$ 5.3 percent (range $-4.2 \%$ to $+14.2 \%$ ) when the limb was contracted versus relaxed was noted. With the liner on, a significant increase in volume of $3.5 \pm 3.3$ percent (range $-1.4 \%$ to $+11.5 \%$ ) was found when the limb was contracted versus relaxed. Neither the mean difference between socket volume and residual limb volume of relaxed limbs (mean $\pm \mathrm{SD}=1.8 \pm 10.1 \%$, range $-15.3 \%$ to $+18.7 \%$ ) nor activated limbs (mean \pm SD $=1.7 \pm$ $11.3 \%$, range $-25.1 \%$ to $+14.1 \%$ ) was significant. Length of time between doffing of the socket and measurement and limb alignment between conditions were not reported. Lilja et al. concluded that the volume changes were large enough that prosthetists should take their cast with the limb relaxed if they want to have stiff coupling between the socket and limb during the stance phase of gait [13]. While the differences between contracted and relaxed residual limbs were outside the measurement error, those between socket volume and limb volume were not (Appendix 6, available online only), consistent with the findings.

\section{Clinical Outcomes Summary}

Overall, limited evidence exists regarding the management of residual limb volume, and the evidence available focuses primarily on adults with transtibial amputation in the early postoperative phase. It is difficult to specify a single best time for definitive socket fitting because of variability in the pattern of postoperative residual limb volume change reported thus far. In general, postoperative volume reduction is initially (first 100-200 days) substantial in persons with transtibial amputation but then decreases with reduced slowing over time. Some data suggest that volume changes beyond 100 to 200 days are more variable. Fewer studies characterize residual limb volume in mature residual limbs over both short- and long-term intervals. Unlike postoperative residual limb volume changes, both increases and decreases in residual limb volume have been reported for mature residual limbs. Some data suggest that limb volume changes immediately after walking and then doffing are greater than those 2 weeks apart. Some data also suggest that mature residual limb volume change varies between individuals based on subject comorbidities.

\section{Group III: What Techniques Have Been Proposed to Accommodate or Control Residual Limb Volume, and What Evidence Exists that They Improve Clinical Outcomes?}

Sixteen studies were reviewed in group III [910,21,24-26,67,71-78] (including one systematic review [17]) that compared different methods of postoperative residual limb volume reduction. Three studies [51,73-74] described the use of early preparatory prostheses that allow volume adjustment, two studies [24-25] evaluated the effect of vacuum-assisted suspension on mature residual limb volume, and three studies [75-76,78] described the use of inflatable inserts to accommodate fluctuations in mature residual limb volume.

Nawijn et al. conducted a systematic review to establish the optimal postamputation management for persons with transtibial amputation, focusing on wound healing, edema reduction, and functional outcome [17]. The authors stated that the reviewed literature was heterogeneous with respect to patient selection, intervention, and outcome measures. Four studies of residual limb volume $[8,67,71-72]$ revealed a trend in favor of rigid and semirigid dressings compared with soft dressings (i.e., elastic bandages) to achieve reduction of residual limb volume in subjects with healed incisions but were inconclusive regarding the effects of early weight-bearing on residual limb healing. Our review includes all the studies identified by Nawijn et al. [17] along with more recent publications. Nawijn et al. [17] recommended that the effect of early weight-bearing should be studied separately from the effect of residual limb dressings, a recommendation that we have followed here.

\section{Effect of Early Postamputation Residual Limb Manage- ment Techniques on Residual Limb Volume}

\section{Removable Rigid Dressings Versus Elastic Ban-} dages. Two studies [72,77] were identified that compared removable rigid dressings with elastic bandages, one of which was included in the review by Nawijn et al. [17] and one that was published more recently. The studies are almost identical with respect to study design, population, interventions, outcome measure, and measurement time points. Both were randomized controlled trials comparing the effectiveness of removable rigid dressing and traditional elastic bandages in reducing residual limb volume in persons with transtibial amputation, with circumference measurements taken at baseline and 2 and 4 weeks postamputation. Residual limb length and circumference measures 
at the tibial tubercle and every $4 \mathrm{~cm}$ distally were used with a frustrum model to calculate residual limb volume. Both sets of authors reported that the elastic bandage group had substantially more variability in residual limb volume than the removable rigid dressing group. While Mueller [72] reported a significant difference in residual limb volume reduction between groups after 4 weeks, Janchai et al. [77] did not (Table 6). Janchai et al. indicated that modifications they made to the removable rigid dressing technique may have affected effectiveness [77]; in particular, cotton socks may not have provided as much compression as the tube socks recommended by Wu et al. [79] in their original description of removable rigid dressings. However, the possibility also exists that the use of absolute units affected Janchai et al.'s ability to detect a statistically significant difference. Both sets of authors reported volume data in cubic centimeters instead of percentage, which while valid, is not easily interpreted. For Mueller, we estimated that residual limb volume changes were $\sim 2$ to 5 percent, near the limit of the resolution capabilities of the circumference measurement technique used (similar to Boonhong [31]) [72]. For Janchai et al., the percentage residual limb volume changes were on the order of 7 to 12 percent, well within the resolution capabilities of the tape measure technique, although variability across the subject population in initial residual limb volume appears to have resulted in variation in the volume change data [77].

\section{Semirigid Dressings Versus Elastic Bandages.}

Two studies compared the use of semirigid dressings and elastic bandages in persons with lower-limb amputation [9-10]. Both studies used volume calculations to estimate the effect of semirigid dressing and elastic bandages on time to fitting of the first prosthesis in persons with lower-limb amputation (mostly transtibial).

Table 6.

Summary of mean volume decrease at 4 weeks postamputation (mean \pm standard deviation).

\begin{tabular}{|c|c|c|c|c|c|}
\hline \multirow{2}{*}{ Study } & \multicolumn{2}{|c|}{ Elastic Bandage } & \multicolumn{2}{|r|}{ RRD } & \multirow{2}{*}{$p$-Value } \\
\hline & $n$ & Volume $\left(\mathrm{cm}^{3}\right)$ & $n$ & Volume $\left(\mathrm{cm}^{3}\right)$ & \\
\hline Mueller [1] & 8 & $31.2 \pm 49.0$ & 8 & $70.7 \pm 21.3$ & $<0.05$ \\
\hline Janchai et al. [2] & 14 & $83.0 \pm 113.1$ & 12 & $79.9 \pm 103.3$ & NS \\
\hline \multicolumn{6}{|c|}{$\begin{array}{l}\text { 1. Mueller MJ. Comparison of removable rigid dressings and elastic bandages } \\
\text { in preprosthetic management of patients with below-knee amputations. } \\
\text { Phys Ther. 1982;62(10):1438-41.[PMID: } 7122702]\end{array}$} \\
\hline \multicolumn{6}{|c|}{$\begin{array}{l}\text { 2. Janchai S, Boonhong J, Tiamprasit J. Comparison of removable rigid dress- } \\
\text { ing and elastic bandage in reducing the residual limb volume of below knee } \\
\text { amputees. J Med Assoc Thai. 2008;91(9):1441-46. [PMID: } 18843876 \text { ] }\end{array}$} \\
\hline \multicolumn{6}{|c|}{ NS = not significant, RRD = removable rigid dressing. } \\
\hline
\end{tabular}

MacLean and Fick conducted a controlled trial of the effect of two postoperative limb management methods (semirigid dressing and soft dressing) on time to readiness for prosthetic fitting in 40 persons with transtibial amputation as a result of PVD [9]. Proximal and distal circumferences (taken at $5 \mathrm{~cm}$ spacing with the medial joint line as the starting point) of the residual limb were made independently by two physical therapists three times a week on alternate days to determine when subjects were ready for prosthetic fitting. Measurements were begun when the incision no longer required a sterile dressing. Criteria for prosthetic readiness included no gaping of the incision and the absence of edema (defined as no further changes in girth of the residual limb on three separate measurements taken every 2 days). Although circumferences were not reported, the authors used the data to calculate Kaplan-Meier survival curves, which suggested that time to readiness for prosthetic fitting was twice as fast in the semirigid dressing group (60 vs 120 days) and that the difference between the two groups was greater beyond 40 days postsurgery.

Wong and Edelstein conducted a randomized control trial of the effect of two postoperative limb management methods (semirigid dressing and soft dressing) on time to readiness for prosthetic fitting in 21 persons with lowerlimb amputation as a result of PVD [10]. The authors used a very similar protocol to MacLean and Fick [9], except that subjects were at least 30 days postamputation when treatment was begun and the cohort included persons with transtibial and transfemoral amputation. Proximal and distal circumference measurements (taken at $5 \mathrm{~cm}$ spacing with the medial joint line as the starting point and moving distally in transtibial amputees; taken at femoral end and moving proximally in transfemoral amputees) of the residual limb were made independently by two physical therapists twice a week to determine when subjects were ready for prosthetic fitting. Therapists used a blank tape to reduce measurement bias. This study accounted for time postdoffing when measurements were taken. Criteria for prosthetic readiness included no opening of the incision larger than $1 \mathrm{~cm}$, cylindrical limb shape confirmed by circumference measurements, stable circumferences for at least 1 week, and subject able to transfer from bed to chair with minimal assistance. Although circumferences were not reported, the authors used the data to calculate Kaplan-Meier survival curves, which suggested that time to readiness for prosthetic fitting was twice as fast in the semirigid dressing group 
(30\% fit within 34 vs 64 days) and that they were fit with a prosthesis sooner after admission to the rehabilitation unit. At 6 month follow-up, more subjects from the semirigid dressing group than the soft dressing group had been fit with a prosthesis and were ambulatory.

Neither of these two studies reported their circumference measurements, making it difficult to assess data quality. Both studies used almost exactly the same protocol, and both reported that semirigid dressings resulted in faster time to prosthetic readiness than elastic bandages, regardless of whether treatment was begun immediately postamputation [9] or 30 days postamputation [10]. Despite the errors inherent in taking circumference measures reliably, the similarity in the findings between studies improves our confidence in the conclusions that use of rigid and semirigid dressings appears to result in faster time to first fitting of a prosthesis than elastic bandages.

Shrinker Socks Versus Elastic Bandages. Manella conducted a randomized controlled trial of 12 persons with transtibial amputation, comparing elastic bandaging to shrinker socks [71]. She used the same measurement technique as Mueller [72] and Janchai et al. [77] and followed subjects for the same amount of time postoperatively. Manella reported an average increase in residual limb volume (from week 1 to week 4) of $16.5 \mathrm{~cm}^{3}$ (not significant) for the elastic bandage group, whereas an average decrease of $63.6 \mathrm{~cm}^{3}$ (significant) was found for the shrinker sock group [71]. The shrinker sock produced a significantly larger decrease in volume after 4 weeks than did elastic bandaging. Although the author reported statistical significance, she did not state what tests were performed. As with Mueller [72] and Janchai et al. [77], the volume data were presented as absolute units (in cubic centimeters) instead of percentages. Converting to percentage residual limb volume, the changes were 1.2 percent for elastic bandaging and 4.2 percent for shrinker socks. This result suggests that the measurement for elastic bandaging was outside the measurement capabilities of the circumference measurement technique, while the results for shrinker socks were near the limit of the measurement capabilities of the circumference measurement technique (Table 1), consistent with the findings regarding significance.

\section{Removable Rigid Dressings Versus Removable} Rigid Dressings + Gel Sock. Graf and Freijah conducted a randomized controlled trial of 16 persons with unilateral transtibial amputation due to vascular insufficiency [21]. They compared standard residual limb care using a removable rigid dressing (control group) with an experimental condition consisting of removable rigid dressing + a polymer gel sock [21]. Limb volume measurements were made at baseline (admission to rehabilitation unit), time of shrinker fitting (determined by physician), and time of casting for the prosthesis (i.e., within the first 3 weeks postamputation). The polymer gel sock was worn only during the period between the initial two measurements. Rate of residual limb volume change was assessed using a cast + water displacement technique as described by Johansson and Oberg [49]. A difference was noted between the two groups in terms of time to each measurement point: the control group was admitted later and spent longer in each stage of the study. It was not clear whether these differences were significant or not. Additionally, two subjects were omitted from the study, but the authors did not report which groups these subjects were from nor how many subjects were ultimately in each group. The authors reported that, overall, both groups demonstrated consistent decreases in residual limb volume, although the variation in individual changes was high. Although use of a polymer gel sock + removable rigid dressing appeared to result in faster reduction in residual limb volume while in use, it did not result in overall changes in the rate of residual limb volume reduction once a shrinker sock was used.

Whirlpool Therapy. Goldberg et al. conducted a before and after trial of seven males with transtibial amputation for whom whirlpool therapy had been prescribed [26]. The purpose of this study was to determine whether or not residual limb edema could be prevented based on the position of the body and residual limb within the whirlpool tank (one tank had the person seated with the residual limb hanging unsupported, one tank had the person seated and the residual limb supported in a horizontal position, and one tank had the person supported in a supine position). A water displacement technique was used to assess residual limb volume after each treatment session. Subjects were treated three times in each of three different whirlpools over a period of 12 days. Order of testing was not described. The authors reported that change in residual limb volume ranged from a $320 \mathrm{~mL}$ decrease to a $390 \mathrm{~mL}$ increase, with no consistency in the direction of change either for any one position or any one patient for three trials in the same tank. The authors reported minimal study details and data, making it difficult to judge the validity of the results and conclusions. 
Effect of Early Preparatory Prostheses with Volume Adjustment on Residual Limb Volume

Three quite different studies were identified that described the use of preparatory prostheses with volume adjustment [67,73-74]. A short, descriptive article by Pinzur et al. described a lightweight, volume- and shapeadaptable preparatory prosthesis constructed of copolymer plastic that could be molded quickly and inexpensively to approximate the smallest volume of an ankle disarticulation residual limb [74]. No data were provided.

A descriptive article by Wilson et al. described an adjustable transtibial socket to be used as a preparatory prosthesis, avoiding the need for several socket changes before residual limb stabilization occurred, or to be used for extended periods during which fluctuation of residual limb volume was difficult to control or shear stresses were a problem [73]. The authors defined the goals of their socket as (1) being custom fitted; (2) using existing prosthetic molding, modification, and fabrication techniques; (3) controlling volume equally or selectively between proximal and distal parts of the residual limb; (4) having normal prosthetic cosmesis; and (5) being light but durable. The proposed socket consisted of a two-part design cinched together with hose clamps. The authors describe fabrication of their proposed socket and mention their fitting experience with six persons with transtibial amputation, but these fittings were not evaluated.

As described previously, Liedberg et al. proposed the tulip limb for temporary use by persons with knee disarticulation or transtibial amputation [67]. The tulip limb consisted of a thin plastic pillow with small plastic pellets that were wrapped around the residual limb and from which the air was evacuated to make it rigid. The limb and pillow were then attached with Velcro bands into a socket made up of four separate sides like the petals of a tulip. The width in all directions could be adjusted. Proximal trim lines extended 10 to $20 \mathrm{~cm}$ above the knee joint line. The socket was attached to an endoskeletal pylon and single axis foot. Although a randomized controlled trial was conducted to compare the effect on residual limb volume of the tulip limb and existing therapy without a limb, minimal study details and data make it difficult to judge the validity of the results and conclusions.

\section{Effect of Vacuum-Assisted Suspension on Mature}

\section{Residual Limb Volume}

Conventional skin suction and vacuum-assisted suspension operate along the same continuum: they both create subatmospheric levels of vacuum. Conventional skin suction involves the person with amputation pushing the residual limb into a slightly undersized socket that incorporates a one-way expulsion valve. This socket causes positive pressure during stance phase and negative pressure during swing phase [80]. Vacuum-assisted suspension refers to use of a mechanical or electrical pump to evacuate air from between the liner-clad residual limb and the socket. Vacuum-assisted suspension applies negative pressure continuously [81]. It does not appear that suction and vacuum-assisted suspension were designed with the intention of reducing residual limb volume change. The literature suggests that the benefits of negative pressure for residual limb volume control were not observed until vacuum-assisted suspension was introduced [22]. It is unclear whether this is because skin suction did not provide noticeable volume control benefits or because these phenomena were simply not reported. Vacuum-assisted suspension was initially assessed on persons with transtibial amputation. Two before and after trials by the same group of authors explored the effect of vacuum-assisted suspension on residual limb volume in persons with unilateral transtibial amputation [24-25].

One study with 11 subjects compared suction and vacuum-assisted suspension to test the hypothesis that the residual limb would maintain volume through increased contact with the liner when vacuum-assisted suspension was used [24]. Subjects had mature residual limbs, had no vascular problems, and were reasonably fit. All subjects wore total surface-bearing sockets with urethane liners and suspension sleeves, with a nylon stocking air wick between the liner and socket. Subjects walked on a treadmill for 30 minutes with and without vacuum at a set speed (1.34 to $1.52 \mathrm{~m} / \mathrm{s}$ ). The suspension condition was randomly assigned and vacuum level was set at $-78 \mathrm{kPa}$. Outcome measures included residual limb volume (assessed with an alginate casting + water displacement method), "pistoning" (assessed with supine X-ray and distraction), and gait symmetry (assessed with video). The authors reported a significant increase in residual limb volume after 30 minutes of walking with vacuum compared with suction (Table 7). Additionally, significantly less pistoning of the tibia and liner was noted, and significantly more stance phase and step length symmetry was found with use of vacuum. The authors concluded that vacuum-assisted suspension drew more fluid into the residual limb than was driven out, which was opposite to suction suspension. They concluded that under vacuum, 
the residual limb of persons with transtibial amputation maintained its volume as the skin remained in greater contact with the liner.

Another study with 11 subjects (although only 7 finished all conditions) assessed the effect on residual limb volume of vacuum-assisted suspension in different socket sizes: undersized socket, neutral, and oversized socket [25]. The authors hypothesized that vacuum-assisted suspension would result in a gain in residual limb volume that exceeded the available socket volume and that the increase in residual limb volume in an oversized socket would cause discomfort, pain, and/or skin redness. Subjects had mature residual limbs, had no vascular problems, and were reasonably fit. Subjects walked on a treadmill at a set speed $(1.25 \mathrm{~m} / \mathrm{s})$ for 18 minutes in each of the three socket conditions with vacuum set at $-78 \mathrm{kPa}$ for all sockets. Sockets were tested in a fixed order beginning with the undersized and ending with the oversized socket, since the oversized socket was hypothesized to cause the most problems. Volume was assessed using an alginate casting + water displacement technique. Postdoffing average residual limb volumes were significantly larger than the volumes available in the undersized, neutral, and oversized sockets. None of the seven subjects who walked in the oversized socket reported discomfort, pain, or redness of the skin.

With regards to standardization, description, and control of the intervention, the authors of these two studies [24-25] were very thorough. However, postdoffing measurement of residual limb volume is an issue. In both studies, residual limb volume was measured with an alginate cast + water displacement technique. The authors state that volume measurements were performed within the first 3 minutes of doffing the socket and that although the possibility exists that the residual limb changed volume during these 3 minutes, they did not examine this issue, given limitations in the technology that existed at the time for measuring repeated instantaneous volumes. They indicated that their concern regarding these measurements was not great because the residual limbs were expected to increase volume during walking and lose volume upon exiting the socket. They believed that if the residual limb lost volume during casting, the conclusion that vacuum-assisted suspension causes a net gain in volume would be understated. However, a more recent study by Zachariah et al. [6] using an optical silhouette scanner to track the residual limb volumes of six persons with transtibial amputation for 35 minutes immediately after doffing the prosthesis suggests that residual limb volume increases by 2.4 to 10.9 percent as soon as the prosthesis is doffed. These results are contradictory to the expectations of Goswami et al. [25]. Therefore, it is unclear how much the time postdoffing before measurements were made affected Goswami et al.'s [25] and Board et al.'s [24] results.

\section{Use of Inflatable Inserts to Accommodate Fluctuations in} Mature Residual Limb Volume

Inflatable inserts are another option for in-socket volume control. Air or fluid added to the inserts is intended to replace fluid that exits the residual limb over time. For example, air-filled bladders may be positioned in the socket with a tube extending through the wall to a pump [76]. The user pumps up the insert to accommodate volume loss. However, most designs have a very small range of volume accommodation [82], limiting their versatility. Another related option is fluid inserts. Fluid inserts are expected to be better than air-filled inserts because the fluid is incompressible. Air-filled inserts are too soft except at moderate to high pressure settings, and they do not expand much over the moderate to high pressure range [76]. These features mean that air-filled inserts cannot perform effectively over a wide range of residual limb fluid volumes, unlike fluid-filled inserts that can accommodate a broad range of limb volume changes. Three studies were reviewed that described the use of in-socket inserts for the management of residual limb volume: two studies described the use of fluid-filled bladders [75-76] and one study described the use of magneto-rheologic (MR) fluid-filled bladders [78].

Table 7.

Summary of mean residual limb volume $(n=11)$ (Board WJ, Street GM, Caspers C. A comparison of trans-tibial amputee suction and vacuum socket conditions. Prosthet Orthot Int. 2001;25(3):202-9. [PMID: 11860094]

DOI:10.1080/03093640108726603).

\begin{tabular}{lccrc}
\hline Condition & $\begin{array}{c}\text { Initial Residual } \\
\text { Limb Volume }(\mathbf{m L})\end{array}$ & $\begin{array}{c}\text { Postdoffing Residual } \\
\text { Limb Volume }(\mathbf{m L})\end{array}$ & $\begin{array}{c}\text { \% Change in Residual } \\
\text { Limb Volume }\end{array}$ & $\begin{array}{c}\boldsymbol{p} \text {-Value } \\
\text { Suction }\end{array}$ \\
Vacuum & 770 & 719 & -6.6 & $<0.001$ \\
\hline \hline
\end{tabular}


A smart variable-geometry socket was described by Greenwald et al. [75] to help maintain socket fit when residual limb volume fluctuations occur. The proposed system had three main components: a fluid reservoir, a mechanical control circuit, and multiple discrete bladders located inside the socket. Power was derived from the user by taking advantage of the cyclic pumping that occurs during gait (compression during stance and suction during swing) to move fluid in and out of the bladders. Number and distribution of bladders was at the discretion of the prosthetist, and maximal pressure was set by the prosthetist to the patient's comfort level. How these design criteria were to be arrived at was not clear from the article. The authors acknowledged that limited data are available that quantify diurnal residual limb volume fluctuations and that the amount of fluctuation varies greatly among individuals with amputation. A continuous slow leak from the bladders and an additional regulator ensured that pressure dropped below the ischemic limit during periods of inactivity. The authors assessed their system in one person with transfemoral amputation and reported that the average daily maximum volume variation over a 20 -day period was $40 \mathrm{~mL}$ in a $1,500 \mathrm{~mL}$ socket $(\sim 2.7 \%)$.

Sanders et al. investigated the effect of socketmounted fluid-filled inserts on socket pressure and shear stress in two males with transtibial amputation, one without comorbidity and one diabetic [76]. Teardrop-shaped polyurethane bladders were taped to the inner wall of slightly oversized patella-tendon bearing sockets, positioned posterior laterally and medially over the gastrocnemius and soleus muscles. A tube exited the socket proximally to allow for fluid to be added or removed. A syringe was used to control the fluid input into the bladders. Thirteen transducers were mounted through holes in the socket wall to measure pressure and shear stresses. Data for a series of insert volumes were collected for the middle 10 to 12 steps of a 16-step trial at self-selected walking speed for increments within each subjects' range of tolerable fluid levels. Fluid additions caused interface pressure increases of 33.1 percent on one subject and 18.1 percent on a second subject. Interface resultant shear stresses increased 60.4 percent on the first subject and 41.5 percent on the second subject. Interface pressure and shear stress changes induced by filling the bladders were larger than step-to-step variability within a fluid level. The authors concluded that, in general, stresses at the transducer sites increased with added fluid and subjects preferred relatively high fluid volume settings, possibly because high fluid volume settings reduced the shear:pressure ratio. Definitive insights were precluded by the limited sample size and short-term data collection session for each volume setting.

A socket with MR fluid-filled bags that can alter the rigidity of the socket to accommodate dynamic movement of the residual limb was proposed by Ogawa et al. [78]. The properties of the inner socket materials were varied with changes in dynamic forces so that contact pressures were adjusted. Socket volume was altered by moving MR fluid as required between the bladders and a reservoir. Socket pressure was controlled through changing the viscosity of the MR fluid by varying the magnetic field strength with magnets mounted on the exterior of the socket. Viscosity ranged from 7 to $18 \mathrm{MPa} / \mathrm{s}$ (average: $12.5 \mathrm{MPa} / \mathrm{s}$ ). An experiment was conducted using nondisabled male subjects (sample size was not reported) to determine the upper bound for pressure that would not cause pain. MR-filled bladders were used to create three different levels of pressure (38 kPa, $63 \mathrm{kPa}$, and $127 \mathrm{kPa}$ ) over 17 locations on the shank (on locations corresponding to a typical transtibial residual limb). How pressure was applied to the nondisabled limb was not clearly explained. Pain was recorded using a visual analog scale. The authors reported that sensitivity to pain was higher posteriorly than anteriorly, with $50 \mathrm{kPa}$ tolerated in the popliteal fossa and $120 \mathrm{kPa}$ at the patella tendon. The authors also assessed their system in one person with transtibial amputation and reported how much pain the subject experienced when the pressure from the MR fluid-filled bags was adjusted to patient tolerances during standing and stepping. Pain results for discrete locations on the residual limb were compared with a total-surfacebearing socket. It was not clear whether the same socket was used for both conditions.

\section{Volume Control Summary}

While a variety of techniques have been proposed to control or accommodate residual limb volume, evidence regarding their effectiveness is limited. Limited evidence exists that rigid and semirigid dressings control postoperative residual limb edema better than elastic bandages in persons with transtibial amputation. Other postoperative management techniques such as early ambulation and the use of preparatory prostheses with volume adjustment have not been studied sufficiently to render a conclusion regarding their effectiveness. Investigations on the use of 
vacuum-assisted suspension to control residual limb volume and inflatable socket inserts to accommodate residual limb volume changes in mature residual limbs are also limited.

\section{DISCUSSION}

Table 8 provides a summary of the outcome statements resulting from this review, including the number of studies contributing to the statement and the level of confidence we have in the statement based on the overall quality of the pertinent studies. We jointly considered the "ranking" based on study design and the "level of confidence" we as reviewers had in the outcomes reported (giving due consideration to the impact of methodological problems). The level of confidence was determined as described by Dillon et al. [83]: each article that contributed to our understanding of a particular outcome was rated according to an adjective-driven scale describing the level of confidence, and then the overall level of confidence for the group of articles was synthesized for each outcome statement.

\section{What Technique Should Be Used for Residual Limb Volume Measurement?}

The technique depends on the purpose for which the researcher or practitioner intends to use the measurement. Circumference measurements with a frustrum or cylindrical model [30-31] are practical and probably sufficiently robust for clinical assessment of macroscopic residual limb volume change, i.e., in cases where the limited resolution and time scale of the technique are not problematic. For example, to investigate volume change in the intermediate recovery stage after surgery, we can use this technique to find out whether the individual is still reducing in residual limb volume and thus is not ready to be fit with a definitive socket. However, the technique is not robust enough to identify when residual limb volume has stabilized. For individuals past the intermediate recovery stage and recently in a definitive socket, circumference measurements from the time of limb fitting to a subsequent measurement weeks later might help identify the need for a new socket. In other words, circumferential measurements with a frustrum or cylindrical model are only effective for measurement of relatively large limb volume changes. We estimate that changes as low as 5 percent can be resolved with a high- quality and consistent measurement technique. Resolution depends strongly on the procedure used.

Results are less prone to error if a few simple steps are taken when performing these measurements. The knee should be put in a consistent degree of extension from one measurement to the next. Extreme care must be taken to ensure the position of the tape measure is consistent from one measurement session to the next. Ink markers can be helpful if they do not rub off between sessions and are placed on areas of the skin that do not displace easily. A consistent tension should be put on the tape measure, and use of a spring-loaded tape measure is advised. A spring-loaded tape measure was deemed slightly more consistent than a standard tape on transtibial limbs covered with gel liners [84], but the influence on actual residual limbs has yet to be tested. Patient activity before doffing the prosthesis should be consistent from one session to the next (preferably sitting or supine for 10 minutes), and the measurements should be taken at a short and consistent time after doffing and at about the same time of day.

Further investigations are needed to determine whether the method using circumference measurements and a cylindrical or frustrum model can be simplified without loss of accuracy, thereby reducing the time needed to conduct the measurement. For example, do results calculated as "percentage residual limb volume change" instead of an absolute difference (in cubic centimeters) reduce variability in the measurement within the population? Is one measurement at $4 \mathrm{~cm}$ below the patellar tendon sufficient for clinical volume change monitoring, or do several measurements at $4 \mathrm{~cm}$ intervals down the limb need to be conducted? Does adding the distal tip of the limb to the model [30] enhance clinical accuracy of the technique for volume change assessment purposes?

Monitoring sock-ply is another common means of gaining insight into residual limb volume change. An increase in the need to add socks suggests substantial limb volume reduction and the need for a new prosthetic socket. Indeed, this method was used [11,14] to estimate what residual limb volume change necessitated a new socket. While some attempts were made in the United States by the Department of Veterans Affairs to standardize wool and cotton sock-ply, it is unclear how widely applied these standards are, especially internationally [85-86]. Further, the effects of compressing the sock within the socket and the effects of wear on sock thickness need investigation. 
Table 8.

Outcome statements regarding residual limb volume.

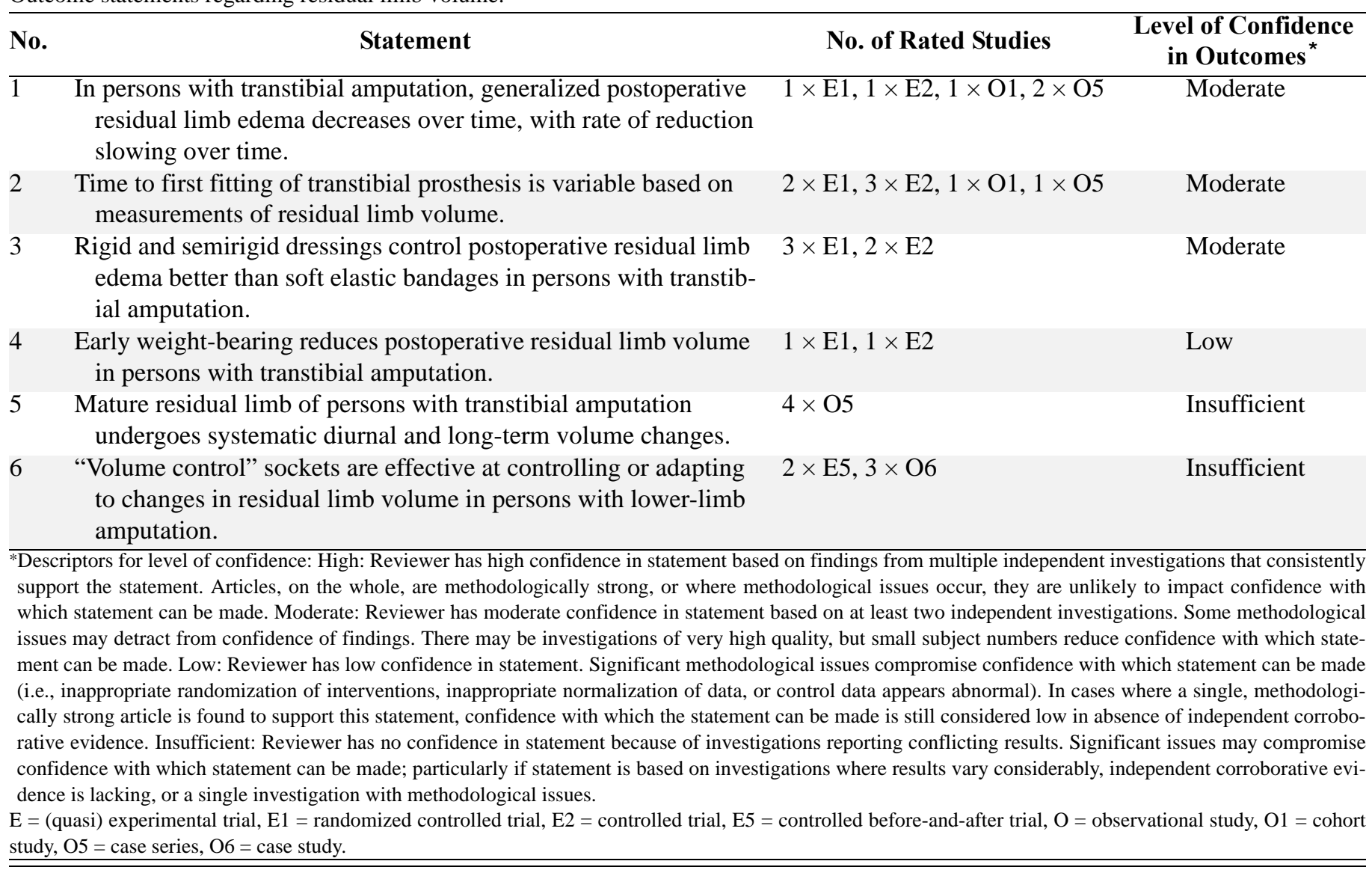

The next step up in volume measurement technique in terms of greater sophistication is water displacement. This method is more accurate than circumference-based assessments in that residual limb volume is measured directly rather than being computed from a model. Like circumferential measurements, water displacement measurements must be taken carefully. For example, the water must be brought up to the same height for each measurement. Most importantly, a lot of people move during measurement, which limits effective measurement with water displacement to select individuals who are capable of holding extremely still during the time needed to take the measurement. For most individuals, this technique would probably not work well to characterize residual limb volume changes over the course of a day.

Noncontact scanners (optical, laser) have good potential for the measurement of residual limb volume change. They are typically used to collect residual limb shape data for socket design, and if they could be configured to assess residual limb volume change, then the practitioner could easily implement this tool in the clinic. However, Geil determined that in 2007, noncontact scanners were no more accurate than caliper measurements for measuring distances [52]. Part of the reason for this finding is that noncontact scanners record a radial measurement as opposed to a circumferential or tangential measurement. Thus, volume measurement error in scanners is proportional to the square of the limb radius as opposed to the limb radius. At the time of Geil's study, noncontact scanners offered no advantage over circumference measurements for assessment of residual limb volume change [52]. If they were made more accurate and they accommodated for limb movement during scanning, then noncontact scanners might provide more useful insight in clinical practice than was previously achieved. It is then possible that noncontact scanners could help us identify when an individual needs a particular treatment to stabilize residual limb volume. Scanners need to better align results from different scans so that comparisons over time can be made and scans 
acquired quickly so as to not be so sensitive to residual limb volume increase after doffing of the socket. The relevance of time postdoffing and its effect on the measurement is clear from Zachariah et al.'s [6] report that volume increases upon socket removal (median of 6.0\%) were much larger than volume changes between sessions 2 weeks apart (median of 2.1\%), with rate of increase highest immediately upon socket removal and decreasing over time. Optical scanners $[6,29,39]$ already overcome the limb shape alignment and image acquisition time issues, but laser scanners do not. To evaluate a scanner's sensitivity to limb movement, developers should conduct repeatability evaluations on subjects 30 minutes after doffing [6] or on limb replicas moved during scanning to simulate subject movement [38]. Once noncontact scanners are made capable of accurate residual limb volume assessment, scientifically rigorous investigations will be needed to determine their diagnostic capability and whether they are effective at improving the outcome of clinical care.

SXCT and bioimpedance are the most sensitive methods pursued to date by researchers for the measurement of residual limb volume change and could be useful in the future for diagnostic purposes. However, they are still in a research state and clinically applicable instruments have yet to be developed. Thus, in the short-term, these techniques will provide insight into how individual presentation, prosthetic design, and other variables affect residual limb volume. This insight will be useful in the future toward whatever residual limb volume measurement techniques emerge as viable for clinical use and should provide evidence to establish best practices for limb volume management, information sorely needed by clinical care providers.

Further, because SXCT and bioimpedance measure volume while the socket is donned, residual limb volume change measurements while wearing different prosthetic designs are possible. However, SXCT is limited to stationary positioning. Bioimpedance can be implemented while an individual ambulates, thus providing insight into changes during standing and activity. Bioimpedance can potentially be used to conduct evidence-based investigations to quantify how different prosthetic designs affect residual limb fluid volume change. Bioimpedance should be capable of identifying whether and how in-socket and postdoffing residual limb fluid volumes are related and thus provide insight into the utility of noncontact scanners for residual limb volume assessment.

\section{What Is the "Natural History" of Postamputation Residual Limb Volume Change?}

Based on five studies of low to moderate quality, with quality assessment based on the AAOP Evidence Report Guidelines described in the "Methods" (p. 951), we have moderate confidence that in persons with transtibial amputation, generalized residual limb edema decreases over time, with the rate of reduction slowing over time. Confidence in this statement was decreased by inconsistent results across studies and relatively poor reliability of measurements. Four studies [11,14,67-68] reported decreased volume over 12, 14, and 23 weeks, while two studies reported less consistent results over slightly longer follow-up periods (28 and 48 weeks, respectively) $[8,14]$. Magnitude of decrease ranged from 1.1 to 35 percent.

A lot of variability exists in the pattern of postoperative residual limb volume change, making it difficult to specify a single best time for definitive socket fitting. No consistent pattern of residual limb volume change has been found across persons with transtibial amputation, and virtually no literature is available on persons with transfemoral amputation. Multiple factors can influence residual limb volume change. For example, an individual's weight changes [14] and changes in the muscle makeup [69] are potentially important. Atrophy of the muscles will reduce residual limb volume, but increases in subcutaneous fat for inactive individuals and muscle tone for active individuals (for muscles sutured under tension) will increase it.

The conclusion from this review is that research to date suggests that postoperative residual limb volume change and the time to stabilization are not easily predicted. It is possible that an analytical model could be developed that systematically considers influential variables, both those already investigated to date and new ones, and that volume changes could be predicted on a subject by subject basis from measurements of these variables. But it is clear that such a model would need to consider a number of factors related to the individual, prosthesis, and environment. Such a model presents substantial challenges for investigators.

\section{When Do Residual Limb Volume Changes Become Less Pronounced?}

Based on six studies of mostly moderate quality, we have moderate confidence in the statement that time to first fitting of a transtibial prosthesis is variable based on 
measurements of residual limb volume [8-11,14,67]. Confidence in this statement was attenuated by the minimal data reported in these studies. Time to fitting of a prosthetic limb in persons with transtibial amputation ranged from 34 to 120 days postamputation [9-10]. Predictions based on tracking residual limb volume and estimating goodness of socket fit suggested longer optimal fitting times, between 100 and 150 days postamputation $[11,14]$.

The literature indicates that no particular postoperative residual limb volume change exists at which a prosthetic socket should be made. However, because a slowing in residual limb volume change over time was noted, limb volume change per unit time (per week or per month, for example) could be useful for determining when a consistent residual limb volume is being approached. However, currently available measurement techniques (anthropometric, water displacement, noncontact scanners) do not have the necessary combination of speed and measurement accuracy for this specific an assessment. Furthermore, it is not yet known how such instruments might be effectively implemented in clinical practice. This topic is a potentially important area of research. Because diurnal volume changes have been recorded on the order of 2 percent [12] and we know that diurnal changes require compensatory actions by many individuals (e.g., managing number of socks [87]), a technique with a resolution of at least 2 percent is needed when using residual limb volume change to identify an appropriate time when consistent socket fit may be achieved. Additional research will determine whether individuals undergo substantial residual limb volume changes after the first prosthetic socket is fit as a result of their enhanced activity, a phenomenon expected based on clinical experience. This information may help to address the struggle currently faced by prosthetists with regards to when to introduce volume-sensitive sockets, such as those using vacuum-assisted suspension, rather than relatively more forgiving volume designs, such as locking liners or suction sockets.

\section{Can Postoperative Residual Limb Management Decrease Residual Limb Volume?}

Based on four experimental studies of mostly moderate quality that compared elastic bandages with rigid or semirigid dressings [9-10,72,77], we have moderate confidence that rigid and semirigid dressings control postoperative residual limb edema better than elastic bandages in persons with transtibial amputation. Confidence in these results was curtailed somewhat by inconsistent significance of results, inferred volume change, and minimal data reporting in some studies. Additional insights into the effectiveness of other postoperative residual limb management techniques cannot be made at this time because comparisons among other techniques have not been reported.

\section{Can Early Ambulation Decrease Residual Limb Volume?}

Based on two experimental studies of low quality that assessed the effect of early ambulation on residual limb volume in persons with transtibial amputation $[8,67]$, we found insufficient evidence that early ambulation decreases residual limb volume faster or to a greater extent than waiting to ambulate. Our assessment concurs with the review by Nawijn et al. [17] that results regarding the effect of early ambulation are inconclusive. Both studies we reviewed were considered to be low in quality because of minimal data reported [67] and confounding effects of residual limb management and ambulation [8].

\section{For an Amputee Wearing a Definitive Socket, How Much Residual Limb Volume Change Causes Problems with Fit?}

Based on four observational studies [6-7,12,14], we found insufficient evidence to characterize residual limb volume change in mature residual limbs over both shortand long-term intervals. One diurnal investigation [12] was of limited scope in that the median absolute volume change $(0.4 \%)$ was within the range of measurement error $(0.1 \%-1.0 \%)$. Only approximately one-fourth of the diurnal absolute volume changes measured were $>1.0$ percent. A second study [7] that used a more sensitive modality showed median absolute fluid volume changes $(0.5 \%)$ outside the measurement error, but changes within a 5-minute walking period as opposed to between sessions were quantified. Median absolute volume changes for two long-term studies (2.1\% [6], 1.3\% [12]) and mean change for a different study (12\%) [14] were outside measurement errors, but inconsistent durations were assessed ( 2 weeks, 5 to 25 weeks, and $>1$ year), making comparisons between studies difficult. Longterm residual limb volume changes ranged from -2.0 to +12.6 percent [6], -4.4 to +4.9 percent [12], and 5 to 12 percent [14]. One study had methodological issues 
since the interval over which volume change was measured was not reported [14].

The results available on volume change in mature residual limbs suggest that for subjects who had their amputation as a result of traumatic injury, absolute shortterm (within day) changes were lower than absolute longterm (2 week to $>1$ year) changes [7,12]. It is unknown whether this would be true for diseased subjects, and this topic is an area of needed investigation. Clinically, it is often diseased subjects who must make accommodations to their prosthesis for short-term residual limb volume fluctuations. Sanders et al. suggested that the health of the individual affected residual limb fluid volume change [7]. A final point is that unlike postoperative residual limbs that tend to decrease in limb volume, in mature residual limbs both increases and decreases have been reported [6-7]. This result suggests that in future investigations, the influence of subject-specific characteristics on mature residual limb volume change should be considered.

The establishment of criteria for what residual limb volume change results in poor socket fit has emerged mainly from observations of sock-ply change, where sock thickness is converted to a percentage residual limb volume $[11,14]$. Clinical observation of transition from a "good" socket fit to an "acceptable" socket fit occurs at $\sim 5$ percent volume loss and 2.5 percent volume gain, while an "unacceptable" socket fit occurs at $~ 10$ percent volume loss and 5.0 percent volume gain [14]. These results assume that the socket fit at the outset is optimal. Residual limb volume changes that are less than these percentages might induce a poor fit if fit at the outset of measurement is borderline. An important need of future research is to establish a greater database of residual limb volume change data and to conduct evidenced-based studies of how residual limb volume change relates to prosthetic socket fit, comfort, and quality of life. This information would provide quantitative metrics for acceptable socket fit, and help promote quantitative fitbased standards as opposed to time-based standards.

\section{What Are Merits of "Volume Control" Sockets?}

Insufficient evidence is available regarding the effectiveness of sockets that control for or adapt to residual limb volume changes [24-26,75-76,78]. Most of the literature on "volume control" sockets is descriptive [73-74]. Where evaluations have been attempted, they are of limited scope
[76], tempered by methodological issues [75,78], or lack independent corroborative evidence [24-25].

\section{Adjustable Sockets During Early Postoperative Phase}

Adjustable sockets for use during the early postoperative phase have been described [73], but evidence as to whether or not they stabilize residual limb volume is lacking as quantitative studies have yet to be conducted. One challenge to this type of research is that instrumentation to measure these small residual limb volume changes is just beginning to emerge. As instrumentation advances, we may discover more. Practitioners need evidence-based studies of how to best implement adjustable sockets and the criteria on which to base adjustment.

\section{Air-Filled Socket Inserts}

Large air-filled socket inserts do not accommodate a very wide range of residual limb volume change [82]. They have a narrow dynamic range because air is compressible and thus reduces volume in proportion to the pressure within the insert. The properties of air-filled inserts are largely dictated by the mechanical properties of the covering material. When inflated to a high pressure, large air-filled inserts are essentially a stiff balloon within the socket. One company has overcome this limitation by using a very elastic rubber material and creating sockets with multiple locations on the inside surface for the insert to compress the residual limb (Pump It Up!, Amputee Treatment Center; Batavia, New York). Fabrication is very labor intensive, and our review did not locate any published reports demonstrating the effectiveness of this design. Nevertheless, this socket and other technology like it should be considered in future research efforts.

\section{Fluid-Filled Socket Inserts}

Fluid-filled inserts overcome the small dynamic range of air-filled inserts. Because liquid is essentially incompressible, a linear relationship is found between fluid volume in the inserts and reduction in socket volume. In a case study, fluid-filled inserts were shown to decrease the shear:pressure ratio at each research subject's preferred pressure setting, though at the expense of increased total interface stress magnitude [76]. Further investigations are needed to understand how fluid-filled inserts affect residual limb soft tissues and the outcome of clinical care. Proof of concept has been demonstrated using MR fluidfilled inserts [78] in that they have been shown to change 
stiffness under an applied electric field. However, clinical performance data are lacking.

Part of the difficulty of creating volume control or adaptive devices is that the specifications they need to achieve are currently unknown [75]. In other words, it is not known what range of volume adaptation is needed or when or where the adaptations should be applied to be most beneficial to the individual with amputation. In short, we are creating a solution to a problem without understanding the problem well enough. As volume change measurement technologies advance, researchers need to fill this knowledge void and provide much needed information to designers of volume control systems.

\section{Vacuum-Assisted Suspension}

In concept, prosthetic sockets with vacuum-assisted suspension should decrease the interstitial fluid pressure during the swing phase of gait in persons with amputation. This interstitial fluid pressure decrease should enhance transport from the arterial system into the interstitial space and decrease transport from the interstitial space into the venous system, resulting in either less of a decrease or an overall increase in residual limb fluid volume. The concept makes sense, and data from the two studies conducted to date are encouraging that vacuumassisted suspension maintains residual limb volume for active persons without comorbidities with transtibial amputation [24-25]. But limitations in the residual limb volume measurement technology used in those studies open up the possibility that vacuum-assisted suspension simply enhanced the speed of limb enlargement postdoffing. No in-socket residual limb volume measurement evidence exists that vacuum-assisted suspension maintains in-socket volume. Vacuum-assisted suspension needs to be tested with in-socket measurement techniques, such as SXCT or bioimpedance, or with scanners that measure very quickly after doffing of the socket. Error from residual limb swelling after doffing must be less than changes measured for the variable of interest.

Of further importance for vacuum-assisted suspension is testing on individuals with comorbidities. For example, individuals with vascular disease may be good candidates for residual limb volume management technologies since they often have fluid-transport problems as part of their disease. Using a prosthesis without volume control, these individuals might require substantial diurnal accommodations to maintain socket fit, which for the insensate individual can be difficult to accomplish effectively. But many clinicians are reluctant to use vacuum-assisted suspension on individuals with comorbidities for fear it may worsen residual limb edema and actually enhance likelihood of residual limb injury. Since the two studies reported in the literature were on nondiseased individuals [24-25], the literature does not guide us. One would expect that the vacuum level would need to be set correctly for each individual. However, what level to set vacuum and how to set it properly remain unknown. This is where research efforts could provide useful insight and be directly relevant to patient care. Researchers should conduct studies to help establish clinical practices for how to select and fit individuals appropriately with volume control systems.

\section{Will Better Residual Limb Volume Measurement Technologies Change Clinical Practice?}

\section{Adaptive Devices}

One use of residual limb volume measurement systems of great potential benefit to prosthetists and patients is a database of residual limb volume change data applicable to the design of adaptive devices, as well as insight into which qualities of the individual with amputation most influence residual limb volume control. In addition, residual limb volume measurement technologies could help in evaluating the effectiveness of one volume management treatment versus another and provide insight into function and quality of life, particularly for individuals with different conditions. For example, does a treatment reduce the individual's adaptive capability or supplement it?

\section{Diagnosis}

Residual limb volume change measurement potentially allows better understanding of the individual's fluid volume control physiology at a level of detail never before possible. As we develop tools to better understand fluid exchanges between the arterial vasculature and interstitial space and between the interstitial space and venous vasculature and how they are influenced, we will better understand each individual's physiology, making it easier to personalize treatment. A challenge to the scientific research community is to pursue this physiological understanding in a manner that is clinically relevant to patient care. 


\section{Prescription}

Potentially, understanding the magnitude and cause of an individual's daily residual limb volume fluctuations will help to optimize prescription. For example, we suspect clinically that vacuum-assisted suspension may not be suitable for all individuals. But who is it suitable for, and can we establish quantitative measures and criteria to select those individuals?

Thus, residual limb volume measurement technologies could potentially change the way prosthetics is practiced, but it will not be until the scientific research community provides information upon which a basis for clinical decision making can be made that this potential will be realized.

\section{Limitations of Systematic Review}

A limitation of this review and potential source of bias was the contribution of the first author to several of the publications included in this review. This potential bias was attenuated to some extent by the contribution of the second author, who has not published on this topic. Both authors endeavored to critically evaluate the literature and report insights in an impartial way. In a small field such as prosthetics, this conflict is difficult to avoid: few individuals have the expertise to critically evaluate a given topic who have not published on that topic.

This review was limited to articles that measured residual limb volume change or calculated it from circumference measures in adults with lower-limb amputation. As such, articles that used indirect assessments of postoperative edema reduction, such as time to healing [79], were excluded. Restricting the review to publications written in English also limited the scope of the review but was consistent with the abilities of the reviewers. An additional and possibly substantial limitation was the exclusion of trade magazines and industry publications, which are a more likely source of descriptions regarding newer technologies and advancements in prosthetics. For example, the Pump It Up! socket was not identified by our review process because no literature on it is present in scientific/medical journals even though it is commercially available. While we did not intend to limit the review to adults with lower-limb amputation, our search did not identify any articles pertaining to children.

As another example of useful insight missed by the traditional systematic review process, suction sockets are a case of researchers starting with a specific reason for a technology (suspension) and then discovering that it was actually relevant to a completely different problem (volume control). Part of the authors' recommendations for future reviews is that such efforts be recognized by the research community and incorporated into our study designs and thinking, even though they are not strictly evidence-based results.

The ability to accurately and reliably measure residual limb volume was an important aspect of this review, hence the inclusion of articles describing various measurement techniques. However, it was not possible to apply the same analysis technique to all articles included in this review, resulting in the need to divide the review into distinct sections. Additionally, the AAOP guidelines [23] that were used to assess quality of studies in group II and III could not be applied to systematic reviews and publications of device description that we designated as "individual opinion" (X2 [Appendix 1, available online only]) for want of a better fitting category. Our review spanned many decades and because study design and reporting of research changes over time, more recent studies may fair better when assessing quality using current standards. However, it does not change the fact that quality ratings identify trials that are more likely to be valid.

\section{CONCLUSIONS}

The purpose of this systematic review was to assess what is known about measurement and management of residual limb volume change in persons with lower-limb amputation. A systematic search of the literature identified 162 publications, with 52 selected for review based on inclusion criteria. Many techniques for the measurement of residual limb volume have been described but clinical use is limited, largely because current techniques lack resolution with regards to small volume changes and most cannot measure in-socket volumes. Those techniques that appear promising are not yet readily available for clinical use. Overall, limited evidence is available regarding the management of residual limb volume and what there is focuses primarily on adults with transtibial amputation in the early postoperative phase. While we can draw some insights from the available research about residual limb volume measurement and management, further research is required. 


\section{ACKNOWLEDGMENTS}

\section{Author Contributions:}

Search and review of articles: J. E. Sanders, S. Fatone.

Drafting of manuscript: J. E. Sanders, S. Fatone.

Financial Disclosures: The authors have declared that no competing interests exist.

Funding/Support: This material was based on work supported by a U.S. Department of Education Grant (H235K080004) to the AAOP.

\section{REFERENCES}

1. Ziegler-Graham K, MacKenzie EJ, Ephraim PL, Travison TG, Brookmeyer R. Estimating the prevalence of limb loss in the United States: 2005 to 2050. Arch Phys Med Rehabil. 2008;89(3):422-29. [PMID: 18295618] DOI:10.1016/j.apmr.2007.11.005

2. Owings MF, Kozak LJ. Ambulatory and inpatient procedures in the United States, 1996. Vital Health Stat 13. 1998; (139):1-119. [PMID: 9866429]

3. Mishra R. Amputation rate for US troops twice that of past wars. Boston Globe. 2005 Dec 9.

4. Fox CJ, Gillespie DL, O’Donnell SD, Rasmussen TE, Goff JM, Johnson CA, Galgon RE, Sarac TP, Rich NM. Contemporary management of wartime vascular trauma. J Vasc Surg. 2005;41(4):638-44. [PMID: 15874928]

DOI:10.1016/j.jvs.2005.01.010

5. Berke G. Post-operative management of the lower extremity amputee: Standards of care. Official findings of the stateof-the-science conferences \#2. J Prosthet Orthot. 2004; 16(3S):6-12.

6. Zachariah SG, Saxena R, Fergason JR, Sanders JE. Shape and volume change in the transtibial residuum over the short term: Preliminary investigation of six subjects. J Rehabil Res Dev. 2004;41(5):683-94. [PMID: 15558398] DOI:10.1682/JRRD.2003.10.0153

7. Sanders JE, Harrison DS, Allyn KJ, Myers TR. Clinical utility of in-socket residual limb volume change measurement: Case study results. Prosthet Orthot Int. 2009;33(4): 378-90. [PMID: 19961297]

DOI:10.3109/03093640903214067

8. Golbranson FL, Wirta RW, Kuncir EJ, Lieber RL, Oishi C. Volume changes occurring in postoperative below-knee residual limbs. J Rehabil Res Dev. 1988;25(2):11-18. [PMID: 3361456]

9. MacLean N, Fick GH. The effect of semirigid dressings on below-knee amputations. Phys Ther. 1994;74(7):668-73. [PMID: 8016199]

10. Wong CK, Edelstein JE. Unna and elastic postoperative dressings: Comparison of their effects on function of adults with amputation and vascular disease. Arch Phys Med
Rehabil. 2000;81(9):1191-98. [PMID: 10987161]

DOI:10.1053/apmr.2000.3780

11. Lilja M, Oberg T. International forum: Proper time for definitive transtibial prosthetic fitting. J Prosthet Orthot. 1997; 9(2):90-95. DOI:10.1097/00008526-199700920-00009

12. Sanders JE, Zachariah SG, Jacobsen AK, Fergason JR. Changes in interface pressures and shear stresses over time on trans-tibial amputee subjects ambulating with prosthetic limbs: Comparison of diurnal and six-month differences. J Biomech. 2005;38(8):1566-73. [PMID: 15958212] DOI:10.1016/j.jbiomech.2004.08.008

13. Lilja M, Johansson S, Oberg T. Relaxed versus activated stump muscles during casting for trans-tibial prostheses. Prosthet Orthot Int. 1999;23(1):13-20. [PMID: 10355639]

14. Fernie GR, Holliday PJ. Volume fluctuations in the residual limbs of lower limb amputees. Arch Phys Med Rehabil. 1982;63(4):162-65. [PMID: 7082139]

15. Boonhong J. Correlation between volumes and circumferences of residual limb in below knee amputees. J Med Assoc Thai. 2006;89(Suppl 3):S1-4. [PMID: 17722302]

16. Singh R, Hunter J, Philip A. Fluid collections in amputee stumps: A common phenomenon. Arch Phys Med Rehabil. 2007;88(5):661-63. [PMID: 17466737]

DOI:10.1016/j.apmr.2007.02.016

17. Nawijn SE, Van der Linde H, Emmelot CH, Hofstad CJ. Stump management after trans-tibial amputation: A systematic review. Prosthet Orthot Int. 2005;29(1):13-26.

[PMID: 16180374]

DOI:10.1080/17461550500066832

18. Hess C. Wound care: Clinical guide. Ambler (PA): Lippincott Williams \& Wilkins; 2005.

19. Taylor AE, Gibson WH, Granger HJ, Guyton AC. The interaction between intracapillary and tissue forces in the overall regulation of interstitial fluid volume. Lymphology. 1973;6(4):192-208. [PMID: 4592823]

20. Smith DG, McFarland LV, Sangeorzan BJ, Reiber GE, Czerniecki JM. Postoperative dressing and management strategies for transtibial amputations: A critical review. J Rehabil Res Dev. 2003;40(3):213-24. [PMID: 14582525]

21. Graf M, Freijah N. Early trans-tibial oedema control using polymer gel socks. Prosthet Orthot Int. 2003;27(3):221-26. [PMID: 14727703]

DOI:10.1080/03093640308726685

22. Street GM. Vacuum suspension and its effects on the limb. Orthopadie Technik. 2006;4:1-7.

23. Hafner B. American Academy of Orthotists and Prosthetists state-of-the-science evidence reports. The Academy Today. 2008;4(2):A4-A8.

24. Board WJ, Street GM, Caspers C. A comparison of transtibial amputee suction and vacuum socket conditions. Prosthet Orthot Int. 2001;25(3):202-9. [PMID: 11860094] DOI:10.1080/03093640108726603 
25. Goswami J, Lynn R, Street G, Harlander M. Walking in a vacuum-assisted socket shifts the stump fluid balance. Prosthet Orthot Int. 2003;27(2):107-13. [PMID: 14571940] DOI:10.1080/03093640308726666

26. Goldberg MJ, Culver JV, Carson JF. Volume changes in below-knee amputation stumps as affected by type of whirpool-tank hydrotherapy. J Am Geriatr Soc. 1968;16(1): 101-105. [PMID: 5634466]

27. Fernie GR, Holliday PJ, Lobb RJ. An instrument for monitoring stump oedema and shrinkage in amputees. Prosthet Orthot Int. 1978;2(2):69-72. [PMID: 364403] DOI:10.3109/03093647809177770

28. Starr TW. A computerized device for the volumetric analysis of the residual limbs of amputees. Bull Prosthet Res. 1980;10-33:98-102. [PMID: 7236952]

29. Commean PK, Smith KE, Cheverud JM, Vannier MW. Precision of surface measurements for below-knee residua. Arch Phys Med Rehabil. 1996;77(5):477-86. [PMID: 8629925] DOI:10.1016/S0003-9993(96)90037-4

30. Krouskop TA, Yalcinkaya M, Muilenberg AL, Holland KC, Zuniga EN. A measurement technique to assess residual limb volume. Orthop Rev. 1979;8:69-77.

31. Boonhong J. Validity and reliability of girth measurement (circumference measurement) for calculating residual limb volume in below-knee amputees. Chula Med J. 2007;51: 77-88.

32. Krouskop TA, Dougherty D, Yalcinkaya MI, Muilenberg A. Measuring the shape and volume of an above-knee stump. Prosthet Orthot Int. 1988;12(3):136-42.

[PMID: 3217243$]$

33. Vannah WM, Drvaric DM, Stand JA, Hastings JA, Slocum JE, Harning DM, Gorton GE. Performance of a continuously sampling hand-held digitizer for residual-limb shape measurement. J Prosthet Orthot. 1997;9(4):157-62. DOI:10.1097/00008526-199700940-00006

34. McGarry T, McHugh B. Evaluation of a contemporary CAD/CAM system. Prosthet Orthot Int. 2005;29(3):22129. [PMID: 16466152] DOI:10.1080/03093640500199497

35. McGarry T, McHugh B. Comparison of the results of four users of a contemporary CAD/CAM system. Prosthet Orthot Int. 2007;31(1):27-35. [PMID: 17365882] DOI:10.1080/03093640600942101

36. McGarry T, McHugh B, Buis A, McKay G. Evaluation of the effect of shape on a contemporary CAD system. Prosthet Orthot Int. 2008;32(2):145-54. [PMID: 18569882] DOI:10.1080/03093640802015920

37. Schreiner RE, Sanders JE. A silhouetting shape sensor for the residual limb of a below-knee amputee. IEEE Trans Rehabil Eng. 1995;3(3):242-53. DOI:10.1109/86.413197

38. Sanders JE, Lee GS. A means to accommodate residual limb movement during optical scanning: A technical note.
IEEE Trans Neural Syst Rehabil Eng. 2008;16(5):505-9. [PMID: 18990654] DOI:10.1109/TNSRE.2008.2003388

39. Commean PK, Smith KE, Vannier MW. Design of a 3-D surface scanner for lower limb prosthetics: A technical note. J Rehabil Res Dev. 1996;33(3):267-78. [PMID: 8823674$]$

40. He P, Xue K, Chen Q, Murka P, Schall S. A PC-based ultrasonic data acquisition system for computer-aided prosthetic socket design. IEEE Trans Rehabil Eng. 1996;4(2):114-19. [PMID: 8798078] DOI:10.1109/86.506408

41. He P, Xue K, Murka P. 3-D imaging of residual limbs using ultrasound. J Rehabil Res Dev. 1997;34(3):269-78. [PMID: 9239619]

42. Smith KE, Vannier MW, Commean PK. Spiral CT volumetry for below-knee residua. IEEE Trans Rehabil Eng. 1995; 3(3):235-41. DOI:10.1109/86.413196

43. Smith KE, Commean PK, Bhatia G, Vannier MW. Validation of spiral CT and optical surface scanning for lower limb stump volumetry. Prosthet Orthot Int. 1995;19:97-107.

44. Smith KE, Commean PK, Vannier MW. Residual-limb shape change: Three-dimensional CT scan measurement and depiction in vivo. Radiology. 1996;200(3):843-50. [PMID: 8756942]

45. Commean PK, Brunsden BS, Smith KE, Vannier MW. Below-knee residual limb shape change measurement and visualization. Arch Phys Med Rehabil. 1998;79(7):772-82. [PMID: 9685090] DOI:10.1016/S0003-9993(98)90355-0

46. Fernie GR, Griggs G, Bartlett S, Lunau K. Shape sensing for computer aided below-knee prosthetic socket design. Prosthet Orthot Int. 1985;9(1):12-16. [PMID: 4000905$]$

47. Oberg K, Kofman J, Karisson A, Lindström B, Siglad G. The CAPOD system-A Scandinavian CAD/CAM system for prosthetic sockets. J Prosthet Orthot. 1989;1(3):139-48. DOI:10.1097/00008526-198904000-00008

48. Lilja M, Oberg T. Volumetric determinations with CAD/ CAM in prosthetics and orthotics: Errors of measurement. J Rehabil Res Dev. 1995;32(2):141-48. [PMID: 7562654]

49. Johansson S, Oberg T. Accuracy and precision of volumetric determinations using two commercial CAD systems for prosthetics: A technical note. J Rehabil Res Dev. 1998; 35(1):27-33. [PMID: 9505250]

50. Buis AW, Condon B, Brennan D, McHugh B, Hadley D. Magnetic resonance imaging technology in transtibial socket research: A pilot study. J Rehabil Res Dev. 2006; 43(7):883-90. [PMID: 17436174]

DOI:10.1682/JRRD.2005.08.0145

51. Sanders JE, Rogers EL, Abrahamson DC. Assessment of residual-limb volume change using bioimpedence. J Rehabil Res Dev. 2007;44(4):525-36. [PMID: 18247249] DOI:10.1682/JRRD.2006.08.0096 
52. Geil MD. Consistency, precision, and accuracy of optical and electromagnetic shape-capturing systems for digital measurement of residual-limb anthropometrics of persons with transtibial amputation. J Rehabil Res Dev. 2007;44(4): 515-24. [PMID: 18247248] DOI:10.1682/JRRD.2006.08.0088

53. De Lorenzo A, Andreoli A, Matthie J, Withers P. Predicting body cell mass with bioimpedance by using theoretical methods: A technological review. J Appl Physiol. 1997; 82(5):1542-58. [PMID: 9134904]

54. Cole KS, Li CL, Bak AF. Electrical analogues for tissues. Exp Neurol. 1969;24(3):459-73. [PMID: 5800962] DOI:10.1016/0014-4886(69)90149-6

55. Grimnes S, Martinsen ØG. Bioimpedance and bioelectricity basics. 2nd ed. Amsterdam (the Netherlands): Elsevier/ Academic; 2008.

56. Miyatani M, Kanehisa H, Masuo Y, Ito M, Fukunaga T. Validity of estimating limb muscle volume by bioelectrical impedance. J Appl Physiol. 2001;91(1):386-94.

[PMID: 11408456]

57. Siconolfi SF, Gretebeck RJ, Wong WW, Pietrzyk RA, Suire SS. Assessing total body and extracellular water from bioelectrical response spectroscopy. J Appl Physiol. 1997; 82(2):704-10. [PMID: 9049756]

58. Zhu F, Sarkar S, Kaitwatcharachai C, Greenwood R, Ronco C, Levin NW. Methods and reproducibility of measurement of resistivity in the calf using regional bioimpedance analysis. Blood Purif. 2003;21(1):131-36. [PMID: 12596759] DOI:10.1159/000067855

59. Kydd WL, Daly CH, Waltz M. Biomechanics of oral mucosa. Front Oral Physiol. 1976;2:108-29. [PMID: 1067215]

60. Vannah WM, Childress DS. An investigation of the threedimensional mechanical response of bulk muscular tissue: Experimental methods and results. In: Spilker RL, Simon BR, editors. Computational methods in bioengineering. New York (NY): ASME; 1988. p. 493-503.

61. Sidles JA, Boone DA, Harlan JS, Burgess EM. Rectification maps: A new method for describing residual limb and socket shapes. J Prosthet Orthot. 1989;1(3):149-53. DOI:10.1097/00008526-198904000-00009

62. Chahande A, Billakanti S, Walsh N. Lower limb shape characterization using feature extraction techniques (noncontact laser scanning). Proceedings of the 16th Annual International Conference of the IEEE Engineering in Medicine and Biology Society; 1994 Nov 3-6; Baltimore, MD. Los Alamitos (CA): IEEE; 1994. p. 482-83.

63. Jimenez D, Darm T, Rogers B, Walsh N. Locating anatomical landmarks for prosthetic design using ensemble neural networks [Internet]. Proceedings of the International Conference on Neural Networks; 1997; Houston, TX. Piscataway (NJ): IEEE; p. 81-87. Available from: http://ieeexplore.ieee.org/xpl/freeabs all.jsp?arnumber=611641.

64. Lemaire ED, Johnson F. A quantitative method for comparing and evaluating manual prosthetic socket modifications. IEEE Trans Rehabil Eng. 1996;4(4):303-9. [PMID: 8973956] DOI:10.1109/86.547931

65. Zachariah SG, Sorenson E, Sanders JE. A method for aligning trans-tibial residual limb shapes so as to identify regions of shape change. IEEE Trans Neural Syst Rehabil Eng. 2005;13(4):551-57. [PMID: 16425837] DOI:10.1109/TNSRE.2005.858459

66. Vannier MW, Commean PK, Brunsden BS, Smith KE. Visualization of prosthesis fit in lower-limb amputees. IEEE Comput Graphics Appl. 1997;17(5):16-29. DOI:10.1109/38.610198

67. Liedberg E, Hommerberg H, Persson BM. Tolerance of early walking with total contact among below-knee amputees-A randomized test. Prosthet Orthot Int. 1983;7(2): 91-95. [PMID: 6622240]

68. Persson BM, Liedberg E. A clinical standard of stump measurement and classification in lower limb amputees. Prosthet Orthot Int. 1983;7(1):17-24. [PMID: 6856447]

69. Lilja M, Hoffmann P, Oberg T. Morphological changes during early trans-tibial prosthetic fitting. Prosthet Orthot Int. 1998;22(2):115-22. [PMID: 9747995]

70. Fernie G, Holliday P, Logg R. An investigation of oedema and shrinkage in the stumps of lower limb amputees [Internet]. Health and Welfare Canada Final Report on Project \# 606-1317-41, West Park Research, West Park Hospital, Toronto, Ontario. Toronto Ontario. 1980. Available from: http://www.amputee-coalition.org/inmotion/mar apr 06/ prosthetic_socks.html.

71. Manella KJ. Comparing the effectiveness of elastic bandages and shrinker socks for lower extremity amputees. Phys Ther. 1981;61(3):334-37. [PMID: 7465627]

72. Mueller MJ. Comparison of removable rigid dressings and elastic bandages in preprosthetic management of patients with below-knee amputations. Phys Ther. 1982;62(10):1438-41. [PMID: 7122702]

73. Wilson AB Jr, Schuch CM, Nitschke RO. A variable volume socket for below-knee prostheses. Clin Prosthet Orthot. 1987;11(1):11-19.

74. Pinzur MS, Angelico JA, Quigley MJ. A volume-adaptable prosthesis for ankle disarticulation. J Prosthet Orthot. 1993; 5(3):77-78. DOI:10.1097/00008526-199307000-00003

75. Greenwald RM, Dean RC, Board WJ. Volume management: Smart Variable Geometry Socket (SVGS) technology for lower-limb prostheses. J Prosthet Orthot. 2003; 15(3):107-12. DOI:10.1097/00008526-200307000-00011

76. Sanders JE, Jacobsen AK, Fergason JR. Effects of fluid insert volume changes on socket pressures and shear stresses: Case studies from two trans-tibial amputee subjects. 
JRRD, Volume 48, Number 8, 2011

Prosthet Orthot Int. 2006;30(3):257-69.

[PMID: 17162516]

DOI:10.1080/03093640600810266

77. Janchai S, Boonhong J, Tiamprasit J. Comparison of removable rigid dressing and elastic bandage in reducing the residual limb volume of below knee amputees. J Med Assoc Thai. 2008;91(9):1441-46. [PMID: 18843876]

78. Ogawa A, Obinata G, Hase K, Dutta A, Nakagawa M. Design of lower limb prosthesis with contact pressure adjustment by MR fluid. Conf Proc IEEE Eng Med Biol Soc. 2008;2008:330-33. [PMID: 19162660]

79. Wu Y, Keagy RD, Krick HJ, Stratigos JS, Betts HB. An innovative removable rigid dressing technique for belowthe-knee amputation. J Bone Joint Surg Am. 1979; 61(5):724-29. [PMID: 457715]

80. Chino N, Pearson JR, Cockrell JL, Mikishko HA, Koepke $\mathrm{GH}$. Negative pressures during swing phase in below-knee prostheses with rubber sleeve suspension. Arch Phys Med Rehabil. 1975;56(1):22-26. [PMID: 1115589]

81. Beil TL, Street GM, Covey SJ. Interface pressures during ambulation using suction and vacuum-assisted prosthetic sockets. J Rehabil Res Dev. 2002;39(6):693-700.

[PMID: 17943671]

82. Sanders JE, Cassisi DV. Mechanical performance of inflatable inserts used in limb prosthetics. J Rehabil Res Dev. 2001;38(4):365-74. [PMID: 11563488]

83. Dillon MP, Fatone S, Hodge MC. Biomechanics of ambulation after partial foot amputation: A systematic literature review. J Prosthet Orthot. 2007;19(8):P2-P61.

DOI:10.1097/JPO.0b013e3180ca8694
84. Geil MD. Consistency and accuracy of measurement of lower-limb amputee anthropometrics. J Rehabil Res Dev. 2005;42(2):131-40. [PMID: 15944877]

DOI:10.1682/JRRD.2004.05.0054

85. Thickness guidelines, single thickness. Kansas City (MO): Knit-Rite, Inc; 1997.

86. A guide to the fitting and selection of prosthetic and orthotic socks and interfaces. Kansas City (MO): Knit-Rite, Inc; 1991.

87. Highsmith MJ, Kahle JT. Prosthetic socks: Simple, relatively inexpensive and critically important [Internet]. InMotion. March/April, 2006;16(2). http://www.amputeecoalition.org/inmotion/mar_apr06/prosthetic_socks.html

Submitted for publication September 30, 2010. Accepted in revised form February 28, 2011.

This article and any supplementary material should be cited as follows:

Sanders JE, Fatone S. Residual limb volume change: Systematic review of measurement and management. J Rehabil Res Dev. 2011;48(8):949-86.

DOI:10.1682/JRRD.2010.09.0189

ResearcherID: Joan Sanders, PhD: E-8204-2011; Stefania Fatone, PhD: E-8490-2011

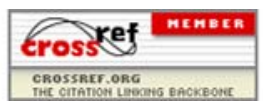

